COMMUNICATIONS IN

ANALYSIS AND GEOMETRY

Volume 5, Number 3, 497-533, 1997

\title{
Geometric Finiteness and the Convergence of Kleinian Groups
}

\author{
EDWARD C. TAYLOR
}

Let $G$ be an abstract finitely generated torsion-free group. Our main result is that if a sequence of discrete, faithful, co-infinite volume representations $\left\{\varphi_{i}\right\}$ of $G$ into $P S L(2, \mathrm{C})$ converges strongly to a geometrically finite representation $\varphi_{\infty}$, then for each large index $i$ the representation $\varphi_{i}$ is itself geometrically finite. This result can be strengthened if the limit representation is convex cocompact. If one assumes convergence of the limit sets and (only) geometric convergence of the groups, in addition to the limit group being convex co-compact, then eventually $\Gamma_{i}$ is convex co-compact for all large index $i$. We finish by sharpening our main result to show that the volume function, when restricted to the convex cores, is continuous if the sequence of representations is converging strongly.

\section{Statement of Results.}

Denote by $\operatorname{Isom}_{+}\left(\mathbf{H}^{3}\right)$ the group of orientation-preserving isometries of $\mathbf{H}^{3}$. A Kleinian group $\Gamma$ is a discrete subgroup of $\operatorname{Isom}_{+}\left(\mathbf{H}^{3}\right)$. If $\Gamma$ is torsion-free, then the quotient manifold $M(\Gamma)=\mathbf{H}^{3} / \Gamma$ is a complete Riemannian manifold with constant sectional curvature -1 . We will restrict ourselves in this paper to the broad class of Kleinian groups that are finitely generated, non-elementary and torsion-free; we also assume the quotient manifold associated to each Kleinian group has infinite hyperbolic volume.

This work is about convergent sequences of Kleinian groups. For the purpose of describing our results, we now present an informal discussion of the different kinds of convergence a sequence of Kleinian groups can exhibit. We say that a sequence of discrete faithful representations $\left\{\varphi_{i}\right\}$ of a group $G$ into $P S L(2, \mathbf{C})$ converges algebraically if, for each $g \in G$, the sequence $\left\{\varphi_{i}(g)\right\}$ converges as Möbius transformations in the compact-open topology. A sequence of Kleinian groups $\left\{\Gamma_{i}\right\}$ converges geometrically to a Kleinian group $\Gamma$ if there exists an increasing sequence of closed balls 
$B_{i} \subset \mathbf{H}^{3}$, such that $B_{i} / \Gamma_{i}$ can be mapped $k_{i}$-quasi-isometrically onto its image in $M(\Gamma)$, and $k_{i} \rightarrow 1$ as $i \rightarrow \infty$. A sequence of discrete faithful representations $\left\{\varphi_{i}: G \rightarrow \Gamma_{i}\right\}$ converging algebraically to $\varphi_{\infty}$, so that the associated sequence $\left\{\Gamma_{i}\right\}$ converges geometrically to $\Gamma=\varphi_{\infty}(G)$, is said to converge strongly. See Section 3 for precise definitions of these concepts.

Every hyperbolic 3-manifold $M(\Gamma)$ (provided $\Gamma$ is non-elementary) contains a minimal convex submanifold, called the convex core, so the inclusion map on this submanifold is a homotopy equivalence (see Section 2). A geometrically finite Kleinian group is one so that, for some $\epsilon>0$, the $\epsilon$ neighborhood of the convex core in the quotient manifold has finite volume. Our main result is:

Main Theorem. Let $\left\{\varphi_{i}\right\}$ be a sequence of discrete faithful representations of a finitely generated, torsion-free abstract group $G$ into $P S L(2, \mathbf{C})$. Suppose the sequence is converging strongly to a representation $\varphi_{\infty}$, so that $\Gamma=\varphi_{\infty}(G)$ is geometrically finite. Then there is an index $\mathcal{I}$, so that for each $i>\mathcal{I}$ the group $\Gamma_{i}=\varphi_{i}(G)$ is geometrically finite.

An immediate consequence of this theorem is the following: A: sequence of degenerate groups converging algebraically to a regular B-group $\Gamma$ on the boundary of a Bers' slice does not converge geometrically to $\Gamma$.

It is well-known that the Main Theorem does not hold in the case where one assumes convergence with respect to either the algebraic structure or the geometric structure alone. However, we do show that if the limit group $\Gamma$ is convex co-compact (i.e. $\Gamma$ is geometrically finite and contains no parabolic subgroups), then the dual assumptions of geometric convergence and convergence of the associated sequence of limit sets are enough to ensure the result.

Theorem (convex co-compact limit). Let $\left\{\Gamma_{i}\right\}$ be a sequence of Kleinian groups converging geometrically to a convex co-compact limit $\Gamma$. Assume the sequence of limit sets $\left\{L_{\Gamma_{i}}\right\}$ is converging in the Hausdorff set metric to the limit set $L_{\Gamma}$. Then there is an index $\mathcal{I}$ so that for each $i>\mathcal{I}$ the group $\Gamma_{i}$ is itself convex co-compact.

In fact, we strengthen this result by showing that under these assumptions then eventually $\Gamma_{i}$ is quasi-conformally conjugate to $\Gamma$. We aliso observe that the assumption that the limit group is convex co-compact is crucial; the statement does not generalize to the broader situation outlined in the Main Theorem. To demonstrate this, we construct (Example 6.2) a sequence of geometrically infinite groups converging geometrically to a geometrically finite limit group (containing a parabolic cyclic subgroup), such that the 
limit sets are converging as well.

Note that in the special case that the limit manifold is geometrically finite and has no cusps, the result above shows that the number of cusps in $M\left(\Gamma_{i}\right)$ for large index $i$ is also identically 0 . More generally, new parabolic subgroups can be formed in the limit of (algebraically, geometrically, or strongly) convergent sequences of Kleinian groups, so there is no hope of generalizing this statement. However, we show in Proposition 5.7 that if the sequence is converging strongly to a geometrically finite group, then eventually the number of non-conjugate parabolic subgroups is non-decreasing in the limit.

As an application of the above mentioned results, we will finish by proving

Theorem. Let $\left\{\varphi_{i}\right\}$ be a sequence of discrete faithful representations of $G$ into $P S L(2, \mathbf{C})$ converging strongly to a representation $\varphi_{\infty}$. Then

$$
\lim \operatorname{vol} C\left(\varphi_{i}(G)\right)=\operatorname{vol} C\left(\varphi_{\infty}(G)\right) .
$$

$C(\Gamma)$ is the smallest convex submanifold in $M(\Gamma)=\mathbf{H}^{3} / \Gamma$ such that the inclusion map is a homotopy equivalence, and vol is the hyperbolic volume in the metric induced by the projection map $\pi: \mathbf{H}^{3} \rightarrow \mathbf{H}^{3} / \Gamma$.

Acknowledgements: The research in this paper constitutes part of a doctoral thesis written at the State University of New York at Stony Brook under the direction of Professor Bernard Maskit. We thank Professor Richard Canary, Professor Maskit and Professor Yair Minsky for detailed conversations concerning this work. We also express our gratitude to the referee for a careful reading of this paper and especially for pointing out a gap in a previous version of the proof of Theorem 6.1.

\section{Basic Definitions and Properties.}

Recall that the group Isom $_{+}\left(\mathrm{H}^{3}\right)$ has a natural identification with $P S L(2, \mathbf{C})$. Thus a Kleinian group $\Gamma$ acts on the Riemann sphere $\widehat{\mathbf{C}}$ as a discrete group of conformal homeomorphisms. This action partitions $\widehat{\mathbf{C}}$ into two disjoint sets: the limit set and the regular set. We define the regular set $\Omega(\Gamma)$ to be the maximal open subset of $\widehat{\mathbf{C}}$ on which $\Gamma$ acts discontinuously. The limit set $L_{\Gamma}$ is $\widehat{\mathbf{C}}-\Omega(\Gamma)$. It is well known that the cardinality of $L_{\Gamma}$ is either $0,1,2$, or $L_{\Gamma}$ is uncountable. If $L_{\Gamma}$ is uncountable then $\Gamma$ is said to be non-elementary; otherwise $\Gamma$ is elementary. The reader is referred to [32] for a discussion of the fundamentals in the theory of Kleinian groups. 
We work in the upper half-space model of hyperbolic 3-space; the hyperbolic distance between points $x, y \in \mathbf{H}^{3}$ is denoted by $\rho(x, y)$. There is a natural geometric model for the action of $\Gamma$ on $\mathbf{H}^{3}$. Choose a point $0 \in \mathbf{H}^{3}$ not fixed by any non-trivial element of $\Gamma$. The Dirichlet fundamental polyhedron based at 0 is the set

$$
P_{0}(\Gamma)=\left\{x \in \mathbf{H}^{3}: \rho(x, 0) \leq \rho(x, \gamma(0)) \forall \gamma \in \Gamma\right\} .
$$

The intersection of the Euclidean closure of $P_{0}(\Gamma)$ with $\partial \mathbf{H}^{3}$ is a furidamental domain (see chapter 2 in [32] for a full definition and examples) for the action of $\Gamma$ on $\Omega(\Gamma)$. We will use a formulation of geometric convergence in terms of Dirichlet polyhedra in Section 6.

We now focus on the geometric and topological properties of hyperbolic 3-manifolds. Results of Margulis give us a complete understanding of the "thin "pieces of any complete hyperbolic 3-manifold $M$. Let inj: $M \rightarrow \mathbf{R}^{+}$ be the continuous function which assigns to $x \in M$ half the length of the shortest homotopically non-trivial curve through $x$. The injectivity radius $\operatorname{inj}(x)$ is the value of this function at the point $x \in M$. For any $\epsilon>0$, define

$$
M^{[\epsilon, \infty)}=\{x \in M: \operatorname{inj}(x) \geq \epsilon\},
$$

and

$$
M^{(0, \epsilon]}=\{x \in M: \operatorname{inj}(x) \leq \epsilon\} .
$$

This is the thick-thin decomposition of $M$. A restricted version of the Margulis Lemma (see [30] and [42]) asserts that if $M$ is a complete hyperbolic 3 -manifold there is a constant $\epsilon_{0}>0$ (the Margulis constant) so that $M^{\left(0, \epsilon_{0}\right]}$ is a disjoint union of pieces homeomorphic to

1. $T^{2} \times[1, \infty)$ where $T^{2}$ is the torus, or

2. $S^{1} \times \mathbf{R} \times[1, \infty)$, or

3. $S^{1} \times D^{2}$, where $D^{2}$ is the closed unit disk.

Items (1) and (2) are known respectively as rank 2 cusps and ranik 1 cusps. Objects of type (3) are neighborhoods of closed geodesics and are called tubes.

Recall that we have made a standing assumption that $I$ ' is nonelementary. We now isolate a submanifold of $M(\Gamma)$ that "contains"the geometry and topology. The convex hull $C H\left(L_{\Gamma}\right)$ is the smallest convex set in $\mathbf{H}^{3}$ containing all geodesics with endpoints in $L_{\Gamma}$. The convex core $C(\Gamma)$ is realized explicitly as the quotient $C H\left(L_{\Gamma}\right) / \Gamma$. Equivalently, it is the 
smallest convex submanifold of $M(\Gamma)$ so that the inclusion map is a homotopy equivalence. Unless the limit set is contained in a geometric circle, the convex core is a 3-manifold with $C^{0}$-boundary. A Kleinian group is geometrically finite if there is an $\epsilon>0$ so that the $\epsilon$-neighborhood of the convex core has finite volume. If the convex core is compact, then $\Gamma$ is said to be convex co-compact. The reader is referred to [11] and [18] for a detailed discussion of the topology and geometry of both convex hulls and convex cores.

The following consequences of a Kleinian group being geometrically finite are those that will be of use to us in the proof of the Main Theorem. Let $\epsilon>0$, and denote by

$$
C^{[\epsilon, \infty)}(\Gamma)=C(\Gamma) \cap M^{[\epsilon, \infty)}(\Gamma)
$$

the $\epsilon$-thick portion of the convex core.

Theorem 2.1. ([34]) Let $\Gamma$ be a geometrically finite group.

1. For any $\epsilon>0$ the submanifold $C^{[\epsilon, \infty)}(\Gamma)$ is compact, and

2. there is a choice of injectivity radius $\epsilon$ sufficiently small so that $M^{(0, \epsilon]}(\Gamma)$ consists only of cusps.

In particular, for small injectivity radius $\epsilon$ (the precise value of $\epsilon$ depends on the geometrically finite group $\Gamma$ ) all closed geodesics in $M(\Gamma)$ have nontrivial intersection with $C^{[\epsilon, \infty)}(\Gamma)$.

A finitely generated Kleinian group $\Gamma$ (respectively $M(\Gamma)$ ) that is not geometrically finite is geometrically infinite. The existence of such groups is a non-trivial matter; see [4], [21] and [31] for details. Theorem 2.2 details the main geometric property of geometrically infinite manifolds that we will be exploiting.

Theorem 2.2. ([7]) Suppose $\Gamma$ is a geometrically infinite Kleinian group and let $x \in M(\Gamma)$ be fixed. Then there exists a sequence of closed geodesics $\left\{\beta_{i}\right\}$ so that

$$
\operatorname{dist}\left(x, \beta_{i}\right)=\min \left\{\rho(x, y): y \in \beta_{i}\right\} \rightarrow \infty
$$

as $i \rightarrow \infty$.

The behaviour of closed geodesics in a geometrically infinite manifold is in marked contrast, as noted by the comment following Theorem 2.1, with the behaviour of closed geodesics in a geometrically finite manifold. 
Theorem 2.2 makes it apparent that no compact region in a geometrically infinite manifold exhibits all of the geometry of the manifold. However we can find a compact set in a geometrically infinite manifold capturing all of the topology. A compact submanifold $N$ of a 3-manifold $M$ is a compact core of $M$ if the inclusion map is a homotopy equivalence. A theorem of Scott [37] asserts if $M$ is an orientable irreducible 3-manifold with finitely generated fundamental group, then $M$ has a compact core.

\section{Algebraic and Geometric Convergence.}

A Kleinian group has an algebraic realization as a group presentation and a geometric realization as the holonomy representation of the fundamental group acting discretely and isometrically on $\mathbf{H}^{3}$. Thus a sequence of Kleinian groups $\left\{\Gamma_{i}\right\}$ can exhibit convergence behaviour with respect to either (or both) the algebraic structure or the geometric structure. We start by considering algebraic convergence. Let $G$ be an abstract finitely generated group with no finite order elements. We say a homomorphism $\varphi: G \rightarrow \Gamma \subset P S L(2, \mathbf{C})$ is discrete and faithful if it is an isomorphism and $\Gamma$ is discrete. A sequence $\left\{\varphi_{i}\right\}$ of discrete faithful representations of $G$ into $P S L(2, \mathrm{C})$ converges algebraically if for each $g \in G$, then $\left\{\varphi_{i}(g)\right\}$ converges in the topology on $P S L(2, \mathbf{C})$ induced by the compact-open topology on the space of Möbius transformations. Let $\varphi_{\infty}(g) \in P S L(2, \mathbf{C})$ be $\varphi_{\infty}(g)=\lim \varphi_{i}(g)$. Jørgensen [23] proved that the set $\Gamma=\left\{\varphi_{\infty}(g): g \in G\right\}$ is a Kleinian group, and there exists an isomorphism $\varphi_{\infty}$ of $G$ realizing $\Gamma$. We refer to $\varphi_{\infty}$ as the algebraic limit, however sometimes we will abuse notation and refer to the group $\Gamma=\varphi_{\infty}(G)$ as the algebraic limit as well.

We recall the definition of geometric convergence. Suppose $T$ is a locally compact topological space, and let $\mathcal{C}(T)$ be the collection of closed subsets of $T$. The geometric topology on $\mathcal{C}(T)$ is characterized in the following manner: A collection of closed sets $\left\{A_{i}\right\}$ in $\mathcal{C}(T)$ converges geometrically to a closed set $A_{\infty}$ if and only if every point $a_{\infty} \in A_{\infty}$ is the limit of a sequence $\left\{a_{i} \in A_{i}\right\}$, and if $\left\{a_{n(i)} \in A_{n(i)}\right\}$ converges to $a$, then $a \in A_{\infty}$. A basic fact (Proposition 3.2.1 in [11]) is that $\mathcal{C}(T)$ is compact in this topology. A sequence of Kleinian groups $\left\{\Gamma_{i}\right\}$ converges geometrically to $\Gamma$ if $\left\{\Gamma_{i}\right\}$ converges to $\Gamma$ in the geometric topology on the space of closed subsets of $\operatorname{PSL}(2, \mathbf{C})$. It was shown in [23] that the geometric limit $\Gamma$ is either elementary or discrete, and if each $\Gamma_{i}$ is torsion-free then the limit group is torsion-free as well. 
There is an equivalent formulation of geometric convergence in terms of the quotient manifolds. From this perspective, one considers the geometric convergence of the quotient manifolds $\left\{M\left(\Gamma_{i}\right)=\mathbf{H}^{3} / \Gamma_{i}\right\}$ to $M(\Gamma)=\mathbf{H}^{3} / \Gamma$. Let $\left\{\Gamma_{i}\right\}$ be a sequence of torsion-free Kleinian groups converging geometrically to a torsion-free group $\Gamma$. Fix a compact set $K_{\infty} \subset M(\Gamma)$. Informally, geometric convergence implies that, far enough out along the sequence, there are compact sets $K_{i} \subset M\left(\Gamma_{i}\right)$ that are "nearly isometric" to $K_{\infty}$.

This version of geometric convergence is now made precise. Fix $0 \in \mathbf{H}^{3}$, and let $B(0, \tilde{r})$ be the closed ball of radius $\tilde{r}$ about 0 . Let $k$ be some constant greater than 1. A $k$-quasi-isometry of a metric space $\left(X, d_{X}\right)$ to a metric space $\left(Y, d_{Y}\right)$ is a mapping $f: X \rightarrow Y$ so that for all $a, b \in X$ then

$$
d_{X}(a, b) / k \leq d_{Y}(f(a), f(b)) \leq k d_{X}(a, b) .
$$

Some authors call such a map " $k$-bi-Lipschitz". A proof of the theorem given below can be found in both [3] and [11], though the statement is taken from [13].

Theorem 3.1. A sequence of torsion-free Kleinian groups $\left\{\Gamma_{i}\right\}$ converges geometrically to a torsion-free Kleinian group $\Gamma$ if and only if there exists a sequence $\left\{\tilde{r}_{i}, k_{i}\right\}$ and there exists a sequence of maps $\tilde{f}_{i}: B\left(0, \tilde{r}_{i}\right) \rightarrow \mathbf{H}^{3}$ such that

1. $\tilde{r}_{i} \rightarrow \infty$ and $k_{i} \rightarrow 1$.

2. The map $\tilde{f}_{i}$ is a diffeomorphism that is $k_{i}$-quasi-isometric onto its image, fixes 0 , and for any compact set $A$ then $\left.\tilde{f}_{i}\right|_{A}$ converges to the identity.

3. The map $\tilde{f}_{i}$ descends to a map $f_{i}: V_{i} \rightarrow M(\Gamma)$, where $V_{i}=B\left(0, \tilde{r}_{i}\right) / \Gamma_{i}$ is a topological submanifold of $M\left(\Gamma_{i}\right)$. Moreover, $f_{i}$ is also a diffeomorphism that is $k_{i}$-quasi-isometric onto its image.

Suppose a sequence of discrete faithful non-elementary representations $\left\{\varphi_{i}\right\}$ has an algebraic limit $\varphi_{\infty}$; let $\Gamma_{A}=\varphi_{\infty}(G)$. By the compactness of $\mathcal{C}(P S L(2, \mathbf{C}))$ in the geometric topology, the sequence $\left\{\Gamma_{i}=\varphi_{i}(G)\right\}$ has a geometrically convergent subsequence. Denote the geometric limit by $\Gamma_{G}$. A basic fact ([42] among other references) is that $\Gamma_{A} \subseteq \Gamma_{G}$. If $\Gamma_{A}=\Gamma_{G}$, then we say the sequence of representations $\left\{\varphi_{i}\right\}$ converges strongly to the representation $\varphi_{\infty}$. If on the other hand $\Gamma_{A}$ is properly contained in $\Gamma_{G}$, then the quotient manifolds $M\left(\Gamma_{A}\right)$ and $M\left(\Gamma_{G}\right)$ will be different both topologically and metrically. Many authors have studied this situation; see both [22] and [26] for explicit examples of this phenomena. 
For the rest of this paper, unless otherwise specified, $\left\{\varphi_{i}\right\}$ is a sequence of discrete faithful non-elementary representations of a finitely generated torsion-free group $G$ into $P S L(2, \mathbf{C})$ converging strongly to a representation $\varphi_{\infty}$. We will maintain the notation $\Gamma_{i}=\varphi_{i}(G)$ and $\Gamma=\varphi_{\infty}(G)$. Denote by $\pi_{i}: \mathbf{H}^{3} \rightarrow \mathbf{H}^{3} / \Gamma_{i}$ the natural projection map; the projection map for the limit group $\Gamma$ is $\pi_{\infty}: \mathbf{H}^{3} \rightarrow \mathbf{H}^{3} / \Gamma$. Recall that for all index $i$, we are assuming that the resulting quotient manifold $M\left(\Gamma_{i}\right)$ has infinite volume.

\section{Outline of the proof of the Main Theorem.}

We give a brief outline of the proof of the Main Theorem in the special case the limit group is convex co-compact. A stronger conclusion will be demonstrated, via a direct argument, using Lemma 5.4 in the next section. In the following, an $\epsilon$ neighborhood of some compact set $K \subset M(\Gamma)$ is denoted by $\mathcal{N}_{\epsilon} K$.

The proof is by contradiction. Suppose there exists a sequence of geometrically infinite representations $\left\{\varphi_{i}: G \rightarrow \Gamma_{i}\right\}$ converging strongly to a convex co-compact representation $\varphi_{\infty}: G \rightarrow \Gamma$. Let $N_{\infty}=\mathcal{N}_{1} C(\Gamma)$; because $\Gamma$ is convex co-compact $N_{\infty}$ is a compact core of $M(\Gamma)$. Choose a point $x_{\infty} \in N_{\infty}$, and fix 0 in $\mathbf{H}^{3}$ so that $0 \in \pi_{\infty}^{-1}\left(x_{\infty}\right)$.

Use the maps $\left\{f_{i}^{-1}\right\}$ defined in Theorem 3.1 to pull $N_{\infty}$ back to $M\left(\Gamma_{i}\right)$. Proposition 3.3 in [13] asserts that the strong convergence of the sequence implies the submanifold $N_{i}=f_{i}^{-1}\left(N_{\infty}\right)$ is a compact core of $M\left(\Gamma_{i}^{\prime}\right)$ for all large $i$. Because the $\Gamma_{i}$ are assumed to be geometrically infinite, we can find (via Theorem 2.2) a sequence of geodesics $\left\{\beta_{i} \in M\left(\Gamma_{i}\right)\right\}$ so that

$$
\operatorname{dist}\left(x_{i}, \beta_{i}\right)=\min \left\{\rho\left(x_{i}, y\right): y \in \beta_{i}\right\} \rightarrow \infty,
$$

where $x_{i} \in N_{i}$ so that $x_{i}=\pi_{i}(0)$. The $N_{i}$ are compact cores, so the geodesic $\beta_{i}$ is homotopic to a curve in $N_{i}$. Using the diffeomorphism $f_{i}$, map this curve in $N_{i}$ to its homotopically non-trivial image in $N_{\infty}$. We then "straighten" the image curve to the geodesic in the homotopy class. Because $\Gamma$ is convex co-compact this geodesic is in $N_{\infty}$ as well.

We again use the maps $\left\{f_{i}^{-1}\right\}$, this time to map the geodesics in $N_{\infty}$ we have just found to curves in $N_{i} \subset M\left(\Gamma_{i}\right)$ homotopic to $\beta_{i}$. These curves are $k_{i}$-quasi-isometric images of closed geodesics with the $k_{i} \rightarrow 1$. Since the image curves are $k_{i}$-quasi-geodesics, each $\beta_{i}$ for large index $i$ is in a fixed distance neighborhood of each quasi-geodesic (see Lemma 5.4). In particular

$$
\beta_{i} \cap N_{i} \neq \emptyset
$$


for all $i$ sufficiently large. This violates (1), and so the desired contradiction is found.

\section{Remarks:}

1.) We show that if a sequence of discrete faithful torsion-free representations is converging strongly to a convex co-compact representation, then eventually $\Gamma_{i}=\varphi_{i}(G)$ is convex co-compact for all $i$ sufficiently large. Note that the argument outlined above only demonstrates that $\Gamma_{i}$ is eventually geometrically finite. A more general result, in the case the limit group is convex co-compact, is known. See the reference to work of Marden in [27] preceding the proof of the Main Theorem (special case).

2.) A limit manifold with cusps presents difficulties. Choose $\epsilon$ sufficiently small so that $M^{(0, \epsilon]}(\Gamma)$ consists solely of cusps. The basic issue is that there are geodesics in a cusped geometrically finite manifold traveling arbitrarily far out some (or all) of the cusps, before returning to the thick part of the convex core. If we follow the argument given above, the image of the curve in $N_{i}$ homotopic to the geodesic $\beta_{i}$ might no longer be homotopic to a closed geodesic contained in $N_{\infty}=\mathcal{N}_{1} C^{[\epsilon, \infty)}(\Gamma)$. Instead, it could be a curve containing geodesic arcs as well as non-geodesic arcs, where each nongeodesic arc wraps around a component in $\partial \mathcal{N}_{1} C^{(0, \epsilon]}(\Gamma)$. If the geodesic arc pieces do not penetrate far enough, and at a steep enough angle, into the interior of the compact core $N_{\infty}$, then we lose control over whether the $k_{i}$-quasi-isometric image of these arcs will intersect $f_{i}^{-1}\left(N_{\infty}\right)$. This problem is handled by further refining our choice of the sequence of closed geodesics $\left\{\beta_{i} \subset M\left(\Gamma_{i}\right)\right\}$.

\section{Proof of the Main Theorem.}

In this section the Main Theorem is proved: We show that a sequence of finitely generated, torsion-free Kleinian groups converging strongly to a geometrically finite group consists of at most a finite number of geometrically infinite groups. This result is proven in two parts: First we prove the result assuming that the limit group is convex co-compact, then we generalize the argument to allow for parabolics. Exploring the convex co-compact case first allows us to consider some of the basic issues without the complications induced by the presence of parabolics.

We need the following two facts. The first result says that the property of being a compact core pulls back under strong convergence. 
Proposition 5.1. (Proposition 3.3 in [13]) Suppose that $N_{\infty}$ is a compact core of $M(\Gamma)$. If $\left\{\varphi_{i}\right\}$ is converging strongly to $\varphi_{\infty}$, then there is an index $\mathcal{I}$ so that $N_{i}=f_{i}^{-1}\left(N_{\infty}\right)$ is a compact core of $M\left(\Gamma_{i}\right)$ for all $i>\mathcal{I}$.

We want to assert both that a $k$-quasi-isometric image of a geodesic segment in $\mathbf{H}^{3}$ is near the segment, and a broken curve consisting of geodesic segments whose geometry is sufficiently "bounded" is close to a geodesic. We begin with some definitions. Let $k \geq 1$, and suppose that $f:[a, b] \rightarrow \mathbf{H}^{3}$ is a smooth embedding that is a $k$-quasi-isometry. The embedding $f$ is called a $k$-quasi-geodesic. For $k \geq 1$ and $\epsilon>0$, a $(k, \epsilon)$-quasi-geodesic is a smooth embedding $f:[a, b] \rightarrow Y$ satisfying

$$
\frac{1}{k} d\left(x_{1}, x_{2}\right)-\frac{\epsilon}{k} \leq d_{Y}\left(f\left(x_{1}\right), f\left(x_{2}\right)\right) \leq k d\left(x_{1}, x_{2}\right)+\epsilon,
$$

where $x_{i=1,2} \in[a, b]$; see [17] chapter 3 . A piece-wise geodesic curve is a curve composed of geodesic arcs. The angle deflection of two arcs in the piecewise geodesic sharing a common endpoint is the angle $\delta<\pi$ supplementary to the angle the arcs make with each other at that endpoint; see Figure 4.2.11 in [11]. Part two of the following theorem states that a piece-wise geodesic and a geodesic are close providing the piece-wise geodesic is not too "broken".

\section{Theorem 5.2}

1. (Mostow [35]) Let $k \geq 1$, and suppose that $f:[a, b] \rightarrow \mathbf{H}^{3}$ is a $k$ quasi-geodesic. Let $\beta$ be the geodesic arc in $\mathbf{H}^{3}$ that connects $f(a)$ to $f(b)$. Then there is a constant $W$ depending only on the distortion constant $k$ (thus not on the particular choice of quasi-geodesic $f$ ) so that a $W$-neighborhood of $\beta$ contains the image of $f . A s k \rightarrow 1$ then $W \rightarrow 0$.

2. ([17] Theorem 11.3.4) Fix an angle deflection $\delta>0$. There are numbers $l_{1}>0, k_{1} \geq 1$ and $\epsilon_{1}>0$, depending on $\delta$, so that the following holds. Let $\alpha$ be a piecewise geodesic in $\mathbf{H}^{3}$, such that each geodesic piece has length at least $l_{1}$, and such that successive pieces mieet at an angle of at least $\pi-\delta$. Let $\alpha$ be parameterized by arc-length. Then $\alpha$ is a $\left(k_{1}, \epsilon_{1}\right)$-quasi-geodesic in $\mathbf{H}^{3}$. In fact $k_{1}$ can be chosen to be any constant greater than 1 ; the closer $k_{1}$ is to 1 the larger $l_{1}$ and $\epsilon_{1}$ must be.

We warm-up for the proof of the Main Theorem by proving it in the special case that the limit representation is convex co-compact. 'This case 
will be an almost direct consequence of a result concerning the geometry of piecewise geodesics (Theorem 5.3 and Lemma 5.4).

There is one issue concerning the proof of Lemma 5.4 that needs to be noted. In particular, there is no canonical choice of a partition of the geodesics inside of the manifolds from which we build our piecewise geodesics. Thus we fix the number of partitions uniformly, and work mainly in the universal cover. The following special version of Theorem 5.2.2 is the formulation that we use in the proof of Lemma 5.4.

Theorem 5.3 Let $\tilde{\alpha}(t)$ be any piecewise geodesic parameterized by arclength having a fixed number of geodesic segments $N$, so that each geodesic segment has a length of at least $L$. Then there is a $\delta>0$, depending on $N$ and $L$, so that the following is true: If the geodesic segments in $\tilde{\alpha}(t)$ with common endpoints have an angle deflection of at most $\delta$, then

$$
\rho(\tilde{\alpha}(t), \tilde{\beta}(t))<L,
$$

where $\tilde{\beta}(t)$ is the geodesic parameterized by arclength that connects the endpoints of $\tilde{\alpha}(t)$.

This fact is reasonably easy to prove: see both Theorem 4.2.10 and Figure 4.2.11 in [11].

Lemma 5.4. Suppose that $\left\{\Gamma_{i}\right\}$ is converging geometrically to $\Gamma$, and let $A_{\infty}$ be any compact subset of $M(\Gamma)$. There exists $a L>0$, and an index $\mathcal{I}$, so that for all $i>\mathcal{I}$ and for any closed geodesic $\beta \subset A_{\infty}$, then

$$
\beta_{i} \subset \mathcal{N}_{L} f_{i}^{-1}(\beta),
$$

where $\beta_{i} \subset M\left(\Gamma_{i}\right)$ is the geodesic in the homotopy class of $f_{i}^{-1}(\beta)$.

Proof. Suppose $A_{i}=f_{i}^{-1}\left(A_{\infty}\right)$ and recall that the injectivity radius function is continuous. By the compactness of $A_{\infty}$, there exists a $L>0$, so that inj $\left.\right|_{A_{\infty}}>2 L$. Since the $f_{i}$ are $k_{i}$-quasi-isometries (with $k_{i} \rightarrow 1$ ), there exists an index $N_{1}$ such that for all index $i>N_{1}$, then $i n j \mid A_{i}>L$.

Our strategy is to build a piecewise geodesic $\tilde{\alpha}_{i}(\beta) \subset \mathbf{H}^{3}$ "near" a lift of $f_{i}^{-1}(\beta)$ for a fixed geodesic $\beta \subset A_{\infty}$. We then deduce from the geometry of this piecewise geodesic that there is a closed geodesic $\beta_{i}$ that is both homotopic to, and in a small neighborhood of, $\alpha_{i}(\beta) \subset M\left(\Gamma_{i}\right)$, where $\alpha_{i}(\beta)$ is the projection under the covering map of $\tilde{\alpha}_{i}(\beta)$. In particular, we will show that

$$
\beta_{i} \subset \mathcal{N}_{L / 2}\left(f_{i}^{-1}(\beta)\right)
$$


The argument is finished by observing that the construction does not depend on the particular choice of $\beta$.

We start by applying Theorem 5.3 to an arbitrary piecewise geodesic having $N$ pieces ( $N$ a fixed number to be specified later). Let $\tilde{\alpha}(t)$ be the arbitrary piecewise geodesic, parameterized by arclength, such that each geodesic segment has length greater than $L / 8$. Let $\tilde{\eta}(t)$ be the geodesic (also parameterized by arclength) connecting the endpoints of $\tilde{\alpha}(t)$. We use Theorem 5.3 to find the angle deflection $\delta$, so that if any two geodesic arc pieces in $\tilde{\alpha}(t)$ sharing a common endpoint meet at an angle of at least $\pi-\delta$, then $\tilde{\eta}(t) \subset \mathcal{N}_{L / 8}(\tilde{\alpha}(t))$.

Fix $a_{\infty} \in A_{\infty}$, and let $0 \in \pi_{\infty}^{-1}\left(a_{\infty}\right)$. Choose a radial length $\ddot{i}>4 L$ so that $A_{\infty} \subset B(0, \tilde{r}) / \Gamma$. Let $\tilde{\beta}$ be the lift of $\beta$ so that $\tilde{\beta} \cap B(0, \tilde{r}) \neq \emptyset$ (observe that we can't assume $\tilde{\beta} \subset B(0, \tilde{r})$, since $\beta$ might have length greater than $\tilde{r}$.)

Since inj $\left.\right|_{A_{\infty}}>2 L$, each closed geodesic in $A_{\infty}$ has a length of at least $4 L$. Thus we can partition $\tilde{\beta}$ into 8 pieces (i.e. $N=8$, where $I N$ is as in Theorem 5.3), so that each geodesic arc in the partition has a length of at least $L / 2$. Since $A_{\infty} \subset B(0, \tilde{r}) / \Gamma$, we can find a collection of geodesic arcs

$$
\left\{\left[\tilde{a}^{k}, \tilde{a}^{k+1}\right]\right\}_{k=1}^{k=8} \subset B(0, \tilde{r})
$$

so this collection projects to $\beta$, and the length of each segment is at least $L / 2$.

By the equivariance of the maps $\tilde{f}_{i}$, there exists a collection of quasigeodesic arcs

$$
\left\{f_{i}^{-1}\left(\left[\tilde{a}_{i}^{k}, \tilde{a}_{i}^{k+1}\right]\right)\right\}_{k=1}^{k=8} \subset B(0, \tilde{r}),
$$

so this collection projects under the covering map to $f_{i}^{-1}(\beta)$. By the choice of $N_{1}$ the length of each $\operatorname{arc} f_{i}^{-1}\left(\left[\tilde{a}_{i}^{k}, \tilde{a}_{i}^{k+1}\right]\right)$ is greater than $L / 4$.

Connect each pair of points $f_{i}^{-1}\left(\tilde{a}_{i}^{k}\right), f_{i}^{-1}\left(\tilde{a}_{i}^{k+1}\right)$ with the geodesic segment defined by the points. For any choice of angle deflection $\delta$, there exists a neighborhood radius $W_{\delta}$ (i.e. the value of $W_{\delta}$ depends on $\delta$ ), so that if each such geodesic segment is in the $W_{\delta}$-neighborhood of the quasi-geodesic arc connecting the same two points then the angle of any two geodesic arcs sharing the same endpoint will be greater than $\pi-\delta$.

Fix such a $W_{\delta} \leq L / 8$ for the angle deflection $\delta$ specified above. Because the $f_{i}^{-1}$ are $k_{i}$-quasi-isometries with $k_{i} \rightarrow 1$, we can use Theorem 5.2 .1 to find an index $N_{2}>N_{1}$ with the following property: The collection of geodesic $\operatorname{arcs} \tilde{\mu}_{i}(\beta) \subset B(0, \tilde{r})$ constructed from connecting the points $f_{i}^{-1}\left(\tilde{a}_{i}^{k}\right)$ to $f_{i}^{-1}\left(\tilde{a}_{i}^{k+1}\right) ; k=1, \ldots, 8$ is in the $W_{\delta}$-neighborhood of $\left\{f_{i}^{-1}\left(\left[\tilde{a}_{i}^{k}, \tilde{a}_{i}^{k+1}\right]\right)\right\}_{k=1}^{k=8}$ for all $i>N_{2}$. Note that this construction is independent of the choice of 
$\beta$; it depends both on the uniformity of the lower bound on the injectivity radius, and the fact that the $f_{i}$ are $k_{i}$-quasi-isometries. Project $\tilde{\mu}_{i}(\beta)$ by the covering map to the piecewise geodesic curve $\alpha_{i}(\beta) \subset \mathcal{N}_{2 L}\left(A_{i}\right)$. Observe that the angle deflection of any two geodesic arcs of $\alpha_{i}(\beta)$ meeting at a common endpoint is no greater than $\delta$, and each segment has length at least $L / 8$.

We form a connected piecewise geodesic by lifting $\alpha_{i}(\beta)$ to $\tilde{\alpha}_{i}(\beta)$, so that $\tilde{\alpha}_{i}(\beta) \cap B(0, \tilde{r}) \neq \emptyset$. Note that $\tilde{\alpha}_{i}(\beta)$ has the same geometry (as a piecewise geodesic) as $\alpha_{i}(\beta)$. Let the collection of endpoints in $\tilde{\alpha}_{i}(\beta)$ be given by $\left\{\tilde{b}_{i}^{k}\right\}_{k=1}^{k=9}$, and let $\tilde{\eta}_{i}(\beta)$ be the geodesic arc connecting $\tilde{b}_{1}$ to $\tilde{b}_{9}$. By our choice of index $N_{2}$, we observe via Theorem 5.3 that for all $i>N_{2}$, then

$$
\tilde{\eta}_{i}(\beta) \subset \mathcal{N}_{L / 8}\left(\tilde{\alpha}_{i}(\beta)\right)
$$

Thus $\tilde{\eta}_{i}(\beta)$ projects to a closed curve $\eta_{i}(\beta) \subset \mathcal{N}_{L}\left(f_{i}^{-1}(\beta)\right)$ that is homotopic to $f_{i}^{-1}(\beta)$. In fact, $\eta_{i}(\beta)$ is by construction a piecewise geodesic with angle deflection at most $\delta$ at $a=\pi_{i}\left(\tilde{b}_{1}\right)=\pi_{i}\left(\tilde{b}_{9}\right)$. Normalize so $\tilde{b}_{1}$ lies on the hyperbolic plane $P=\left\{(x, y, t) \in \mathbf{H}^{3}: x^{2}+y^{2}+t^{2}=1\right\}$. Recall that $\eta_{i}(\beta)$ is formed by factoring the geodesic $\left[\tilde{b}_{1}, \tilde{b}_{9}\right]$ by the action of the group. Since the angle deflection at $a$ is bounded above by $\delta$, we can choose $\delta$ sufficiently small so $\left[\tilde{b}_{1}, \tilde{b}_{9}\right] \subset N_{L / 4}\left(l_{t}\right)$, where $l_{t} \subset \mathbf{H}^{3}$ is the hyperbolic line $\{(0,0, t): t>0\}$. Thus $l_{t}$ projects under the group action $\Gamma_{i}$ to a closed geodesic $\beta_{i}$ in a $L / 4$ neighborhood of $\eta_{i}(\beta)$, finishing the result.

Remark: The choice of using $N=8$ segments in the proof of Lemma 5.4 to construct a piecewise geodesic was arbitrary. When we consider the general case where cusps are present in $M(\Gamma)$, we will observe that there is a natural partition resulting from the geodesics penetrating "deeply" into the cusps.

We now prove the Main Theorem in the special case the limit group is convex co-compact. This result is well-known by deep results of Marden [27]. In fact, Marden showed that the space of convex co-compact representations is open in the algebraic topology. Independently, Thurston [42], Douady-Earle [15] and Riemann [36] proved that each $K$-quasi-conformal deformation of a marked convex co-compact group extends to an equivariant $\tilde{K}$-quasi-isometry in $\mathbf{H}^{3}(\tilde{K} \rightarrow 1$ as $K \rightarrow 1)$. Therefore an algebraically convergent sequence of Kleinian groups, converging to a convex co-compact group $\Gamma$, actually converges strongly.

Our more limited result is included to demonstrate how much easier the proof of the Main Theorem is when there are no parabolic subgroups in the limit group, and also to motivate results in Section 6. 
Main Theorem (special case): Suppose $\left\{\varphi_{i}\right\}$ is a sequence of discrete faithful representations converging strongly to a limit representation $\varphi_{\infty}$. Assume $\Gamma=\varphi_{\infty}(G)$ is convex co-compact. Then there exists an index $\mathcal{I}$ so that for each $i>\mathcal{I}$ the group $\Gamma_{i}=\varphi_{i}(G)$ is convex co-compact.

Proof. We start with an observation. Let $M(\Gamma)$ be a hyperbolic manifold, and suppose there is a compact set $A$ in $M(\Gamma)$ so that every closed geodesic is contained in $A$. Then we claim that there exists a $K>0$ so that $C(\Gamma) \subset$ $\mathcal{N}_{K}(A)$. To show this fix $0 \in \mathbf{H}^{3}$ and form $P_{0}(\Gamma)$. Let $\tilde{C} \subset C H\left(L_{\Gamma}\right) \cap P_{0}(\Gamma)$ be so that $\tilde{C} / \Gamma=C(\Gamma)$. Let $\tilde{A} \subset P_{0}(\Gamma)$ be a compact set so that $\tilde{A} / \Gamma=A$. Clearly $\tilde{C} \cap \tilde{A} \neq \emptyset$.

We claim that $\tilde{C}$ must be compact as well: For suppose not. 'Then the Euclidean closure of $\tilde{C}$ intersect with the Riemann sphere is a non-empty subset of $L_{\Gamma}$. Fix a point in this intersection; there exists a secuence of attracting and repelling fixed points of loxodromic elements converging to this point ([32] Proposition V.E.5.). The axis connecting the fixed points has non-trivial intersection with $\tilde{C}-\tilde{A}$, a contradiction.

Since $\Gamma=\varphi_{\infty}(G)$ is convex co-compact, then $N_{\infty}=\mathcal{N}_{1} C(\Gamma)$ is a compact core of $M(\Gamma)$. The sequence $\left\{\varphi_{i}\right\}$ is converging strongly to $\varphi_{\infty}$, so there is an index $\mathcal{I}_{1}$ such that for all $i>\mathcal{I}_{1}$, then $N_{i}=f_{i}^{-1}\left(N_{\infty}\right)$ is a compact core of $M\left(\Gamma_{i}\right)$. Thus for each closed geodesic $\beta \subset M(\Gamma)$, the curve $\zeta_{i}=f_{i}^{-1}(\beta)$ is both contained in $N_{i}$ and homotopically non-trivial.

Let $\beta_{i}$ be the closed geodesic in $\left[\zeta_{i}\right] \in \pi_{1} M\left(\Gamma_{i}\right)$. Lemma 5.4 implies that there is an index $\mathcal{I}_{2}$ so that $\beta_{i}$ is contained in $N_{i}$ for all $i>\mathcal{I}_{2}$. By the definition of the compact core, $N_{i}$ carries the homotopy of $M\left(\Gamma_{i}\right)$ for all $i>\max \left\{\mathcal{I}_{1}, \mathcal{I}_{2}\right\}$. Thus we have shown that there exists a $K>0$ so that $C\left(\Gamma_{i}\right) \subset \mathcal{N}_{K}\left(N_{i}\right)$ for all large index $i$. In particular, $\Gamma_{i}=\varphi_{i}(G)$ is convex co-compact.

We are ready to prove the Main Theorem in the case that the strong limit $\Gamma$ is geometrically finite and contains at least one conjugacy class of parabolic subgroups. Choose an $\epsilon>0$ so that $M^{(0,2 \epsilon]}(\Gamma)$ consists solely of rank 1 and/or rank 2 cusps. Let $N_{\infty}=C^{[\epsilon, \infty)}(\Gamma)$; observe that $N_{\infty}$ is a compact core of the manifold $M(\Gamma)$. It is important to note that the first part of the following argument holds for any such choice of injectivity radius $\epsilon$; in the course of the proof we make a particular choice of $\epsilon$ so that Theorem 5.2.2 can be invoked. 
Proof of the Main Theorem. The proof is by contradiction: Suppose there exists a sequence of geometrically infinite representations $\left\{\varphi_{i}\right\}$ converging strongly to a geometrically finite limit representation $\varphi_{\infty}$. Fix $0 \in \mathbf{H}^{3}$ and let $x_{i}=\pi_{i}(0)$. We can find, using a diagonalization argument if necessary, a sequence of closed geodesics $\beta_{i} \subset M\left(\Gamma_{i}\right)$ such that $\operatorname{dist}\left(x_{i}, \beta_{i}\right) \rightarrow \infty$. (Recall that $\Gamma_{i}=\varphi_{i}(G)$ and $\Gamma=\varphi_{\infty}(G)$.) Since $N_{\infty}$ is a compact core of $M(\Gamma)$, then for all large index $N_{i}=f_{i}^{-1}\left(N_{\infty}\right)$ is a compact core of $M\left(\Gamma_{i}\right)$ by Proposition 5.1 .

We start with a discussion of certain issues that are not particular to the choice of injectivity radius. Let $\alpha_{i}$ be a curve in $N_{i}$ homotopic to $\beta_{i}$. The curve $\alpha_{i}$ is mapped, via $f_{i}$, to a homotopically non-trivial curve $\widehat{\alpha}_{i}$ in $N_{\infty}$. Denote the geodesic representative in $\left[\widehat{\alpha}_{i}\right] \in \pi_{1} M(\Gamma)$ by $\widehat{\beta}_{i}$. We will refer to $\widehat{\beta}_{i}$ as the push-forward in $N_{\infty}$ of $\beta_{i}$. If the strong limit $\Gamma$ were convex co-compact, then $\widehat{\beta}_{i}$ would be properly contained in $N_{\infty}$. However, since we are assuming the existence of at least one conjugacy class of parabolic elements in $\Gamma$, it is possible the sequence of push-forwards $\left\{\widehat{\beta}_{i}\right\}$ exhibits the property

$$
\operatorname{Dist}\left(x_{\infty}, \widehat{\beta}_{i}\right)=\max \left\{\rho\left(x_{\infty}, y\right): y \in \widehat{\beta}_{i}\right\} \rightarrow \infty
$$

as $i \rightarrow \infty$, where $x_{\infty}=\pi_{\infty}(0)$. If, for our choice of sequence $\left\{\beta_{i} \subset M\left(\Gamma_{i}\right)\right\}$, there exists a $R_{\infty}>0$ such that $\operatorname{Dist}\left(x_{\infty}, \widehat{\beta}_{i}\right)<R_{\infty}$ for all $i$, then the proof of the Main Theorem is completed immediately with an application of Lemma 5.4. Henceforth we assume we are dealing with the situation described by (5.1).

Our first difficulty caused by the presence of cusps in $M(\Gamma)$ is that (infinitely often) the push-forward of $\beta_{i}$ to $N_{\infty}$ might be homotopic to some curve in $\partial N_{\infty} \cap M^{(0, \epsilon]}(\Gamma)$. Because $\Gamma$ is geometrically finite, it contains only finitely many inequivalent conjugacy classes of parabolic elements. Since $\left\{\Gamma_{i}=\varphi_{i}(G)\right\}$ converges strongly to $\Gamma=\varphi_{\infty}(G)$, there exists at most a finite number of closed geodesics in each $M\left(\Gamma_{i}\right)$ that are being pinched down to cusps in the limit manifold. Let $\mathcal{P}=\left\{g_{1}, g_{2}, \ldots, g_{s}\right\}$ be the subset of gen $G$ so that $\varphi_{i}\left(g_{j}\right) \in \Gamma_{i}$ is a loxodromic element being pinched down to a cusp. Let $\beta_{i}^{(j)} \subset M\left(\Gamma_{i}\right)$ be the geodesic associated to $\varphi_{i}\left(g_{j}\right)$. Each such geodesic has a tubular neighborhood of radius $R\left(\beta_{i}^{(j)}\right)$ that is precisely invariant under $\left\langle\varphi_{i}\left(g_{j}\right)\right\rangle$ in $\Gamma_{i}$. Let $R_{i}=\max _{j=1, \ldots, s} R\left(\beta_{i}^{(j)}\right)$ (note that by [10] $\lim _{i \rightarrow \infty} R_{i}=\infty$.) Thus there exists an $R(i) \geq R_{i}$ so if $\beta_{i} \subset M\left(\Gamma_{i}\right)-B\left(x_{i}, R(i)\right)$, then the push-forward of $\beta_{i}$ will not be homotopic to any curve on $\partial N_{\infty} \cap M^{(0, \epsilon]}(\Gamma)$. Since $\Gamma_{i}$ is assumed to be geometrically infinite for all index $i$, such a closed geodesic can be found in each $M\left(\Gamma_{i}\right)$. 
Thus we can replace any geodesic in our original sequence $\left\{\beta_{i}\right\}$, so that the push-forward $\widehat{\beta}_{i}$ is not homotopic to any curve on annular or toroidal boundary components of $N_{\infty}$.

The basic construction is described. Replace the geodesic $\widehat{\beta}_{i}$ with a broken curve $\widehat{\zeta}_{i} \subset N_{\infty}$ homotopic to $\widehat{\beta}_{i}$. Recall the criterion for our choice of injectivity radius: $\epsilon$ is chosen small enough so that $M^{(0,2 \epsilon)}\left(\Gamma^{\prime}\right)$ consists solely of rank 1 and/or rank 2 cusps. Thus each closed geodesic $\beta \subset M(\Gamma)$ has the property that $C^{[\epsilon, \infty)}(\Gamma) \cap \beta \neq \emptyset$. Therefore a "long" closed geodesic goes out some cusp, wrapping around this cusp some number of times, then returns to travel through the $\epsilon$-thick part of the core, possibly going out some other cusp and wrapping around it some number of times, and so forth.

For any such choice of injectivity radius $\epsilon$, we can construct a broken curve $\widehat{\zeta}_{i}$ from $\widehat{\beta}_{i}$ by letting

$$
\widehat{\zeta}_{i} \cap C^{[\epsilon, \infty)}(\Gamma)=\widehat{\beta}_{i} \cap C^{[\epsilon, \infty)}(\Gamma),
$$

and then replacing the remaining segments with non-geodesic arcs that wrap around components in $N_{\infty} \cap C^{(0, \epsilon]}(\Gamma)$. The number of times these nongeodesic arcs are "wrapped" around each component in $N_{\infty} \cap C^{(0, \epsilon]}(\Gamma)$ is the same as the number of times the geodesic wraps around the same component during each visit to $C^{(0, \epsilon)}(\Gamma)$. The observation that $\widehat{\zeta}_{i}$ is homotopic to $\widehat{\beta}_{i}$ is immediate from the fact that $N_{\infty}$ is a compact core, and by the construction.

We emphasize that we will refine this construction by choosing injectivity radii sufficiently small, so as to be able to construct a broken geodesic in $M\left(\Gamma_{i}\right)$ with a desired fixed geometry.

To simplify our description of $\widehat{\zeta}_{i}$, we refer to the non-geodesic pieces as cusp arcs. Let

$$
\widehat{\zeta}_{i}=\widehat{\kappa}_{i}^{1} \cup \widehat{\kappa}_{i}^{2} \cup \ldots \cup \widehat{\kappa}_{i}^{n(i)},
$$

where $\widehat{\kappa}_{i}^{j}: 1 \leq j \leq n(i)$ is either a geodesic arc or a cusp arc. Recall the choice of the sequence $\left\{\beta_{i}: \beta_{i} \subset M\left(\Gamma_{i}\right)\right\}$ has been refined so the pushforward of each geodesic is not homotopic to any curve on the annuli or tori on the boundary of $N_{\infty}$; thus we are assured that for each $i$ there exists at least one $j(i) \in[1, \ldots, n(i)]$ so that $\widehat{\kappa}_{i}^{j(i)}$ is a geodesic arc.

Let $\zeta_{i}=f_{i}^{-1}\left(\widehat{\zeta}_{i}\right)$, and note $\zeta_{i} \in\left[\beta_{i}\right]$ in $\pi_{1} M\left(\Gamma_{i}\right)$ so that $\widehat{\zeta}_{i} \in\left[\widehat{\beta}_{i}\right]$. The broken curve $\zeta_{i} \subset N_{i}$ also has the form

$$
\zeta_{i}=\kappa_{i}^{1} \cup \kappa_{i}^{2} \cup \ldots \cup \kappa_{i}^{n(i)} .
$$

Here the pieces $\kappa_{i}^{j}: 1 \leq j \leq n(i)$ are either 
1. a quasi-isometric image of a geodesic arc, or

2. a quasi-isometric image of a cusp arc.

Following our nomenclature for the segments of $\widehat{\zeta}_{i}$, segments of $\zeta_{i}$ of type (1) are called quasi-geodesic arcs, and segments of type 2 are called quasi-cusp arcs.

Fix an angle deflection $\delta>0$, and let $l_{1}$ be as in Theorem 5.2.2. Recall that the choice of injectivity radius $\epsilon$ was made so that $M^{(0,2 \epsilon]}(\Gamma)$ consists solely of rank 1 and or rank 2 cusps. We will specify two choices of injectivity radii $\epsilon>\epsilon_{l_{1}}>\epsilon_{\delta}$ from which we will build our broken geodesic. In particular, we will consider sequences of closed geodesics $\left\{\beta_{i} \subset M\left(\Gamma_{i}\right)\right\}$ so that dist $\left(x_{i}, \beta_{i}\right) \rightarrow \infty$, and so that for all index $i$ sufficiently large, the push-forwards will have the property that

$$
\widehat{\beta}_{i} \cap M^{\left(0, \epsilon_{\delta}\right)}(\Gamma) \neq \emptyset .
$$

We let $N_{\infty}=C^{\left[\epsilon_{\delta}, \infty\right)}(\Gamma)$, and then build the broken curve $\widehat{\zeta}_{i}$ in the manner explained above, by replacing any piece of $\widehat{\beta}_{i} \cap M^{\left(0, \epsilon_{\delta}\right]}(\Gamma)$ with a cusp arc on the appropriate toroidal or annular boundary component of $N_{\infty}$. By choosing $\epsilon_{l_{1}}$ and $\epsilon_{\delta}$ appropriately, we will construct a piecewise geodesic built from $\zeta_{i}=f_{i}^{-1}\left(\widehat{\zeta}_{i}\right)$ that has angle deflection bounded above by $\delta$, and so the length of any geodesic piece is bounded below by $l_{1}$.

First we bound the length of the geodesic arc pieces. Specifically, we fix a choice of injectivity radius $\epsilon_{l_{1}}<\epsilon$ so that the length of each component of $\widehat{\beta}_{i} \cap C^{[\epsilon, \infty)}(\Gamma)$ is greater than $2 l_{1}$ if $\widehat{\beta}_{i} \cap M^{\left(0, \epsilon_{l_{1}}\right)}(\Gamma) \neq \emptyset$. The choice of $\beta_{i} \subset M\left(\Gamma_{i}\right)$ is refined so in fact $\widehat{\beta}_{i} \cap M^{\left(0, \epsilon_{l_{1}}\right)}(\Gamma) \neq \emptyset$ for all large index $i$. Observe that if we can't find such a choice of sequence then $\widehat{\beta}_{i}$ stays a bounded distance from the basepoint $x_{\infty}$, and therefore the proof of the theorem again would follow from an application of Lemma 5.4.

Temporarily let $N_{\infty}=C^{\left[\epsilon_{l_{1}}, \infty\right)}(\Gamma)$ and let $\widehat{\zeta}_{i}$ be constructed from $\widehat{\beta}_{i}$ so that it consists of geodesic pieces in $N_{\infty}$, and cusp arcs on $\partial C^{(0, \epsilon]}(\Gamma)$ coming from $\widehat{\beta}_{i} \cap M^{\left(0, \epsilon_{l_{1}}\right)}(\Gamma) \neq \emptyset$. Fix $\tilde{R}>4 l_{1}$ so that $N_{i} \subset B(0, \tilde{R}) / \Gamma_{i}$, where $N_{i}=f_{i}^{-1}\left(N_{\infty}\right)$. We can find such a radial length because $\Gamma$ is geometrically finite. Lift $\zeta_{i}=f_{i}^{-1}\left(\widehat{\zeta}_{i}\right)$ to the connected component $\tilde{\zeta}_{i} \subset \mathbf{H}^{3}$ so that $\tilde{\zeta}_{i} \cap B(0, \tilde{R}) \neq \emptyset$. Explicitly, $\tilde{\zeta}_{i}$ consists of quasi-geodesic arcs and quasicusp arcs. We will assemble a piecewise geodesic from $\tilde{\zeta}_{i}$ with the desired geometry (i.e. all segments have length at least $l_{1}$ and the angle deflection is bounded below by $\delta$ ), refining the argument as necessary. Note that each quasi-geodesic arc in $\zeta_{i}$ is the $k_{i}$-quasi-geodesic image of a geodesic arc in $\widehat{\zeta}_{i}$, and in particular each such geodesic arc has a length of at least $2 l_{1}$. 
Replace each quasi-geodesic arc in $\tilde{\zeta}_{i}$ with a geodesic replacement arc: because $f_{i}^{-1}$ are $k_{i}$-quasi-isometries with $k_{i} \rightarrow 1$, then for all index $i$ sufficiently large the geodesic replacement arcs have length at least $l_{1}$. "Unwrap" each quasi-cusp arc in $\tilde{\zeta}_{i}$ and replace each with the geodesic arc that connects its endpoints. We bound the length of the geodesic replacement arcs of the quasi-cusp arcs from below by $l_{1}$. There exists a choice of injectivity radius $\epsilon_{2}<\epsilon_{l_{1}}<\epsilon$, so that if $\widehat{\beta}_{i} \cap C^{\left(0, \epsilon_{2}\right)}(\Gamma) \neq \emptyset$, then the distance the geodesic has traveled in each component of $M^{(0, \epsilon)}(\Gamma)$ from $\partial C^{\left[\epsilon_{1}, \infty\right)}(\Gamma) \cap M^{\left(0, \epsilon_{l_{1}}\right]}(\Gamma)$ to $\partial C^{\left[\epsilon_{2}, \infty\right)}(\Gamma) \cap M^{\left(0, \epsilon_{2}\right]}(\Gamma)$ is at least $2 l_{1}$. Thus eventually, because the $f_{i}$ are $k_{i}$-quasi-isometries with $k_{i} \rightarrow 1$, we deduce that the length of each geodesic replacement arc of a quasi-cusp arc in $\tilde{\zeta}_{i}$ has a length greater tha:n $l_{1}$.

Now we bound the angle deflection. The issue is the angle deflection between the geodesic replacement of a quasi-cusp arc and the geodesic replacement quasi-geodesic arc sharing a common endpoint. In the Figure 1, $\Theta<\pi$ is the angle that a quasi-cusp arc makes with a quasi-geodesic arc sharing the same endpoint in $\zeta_{i}$.

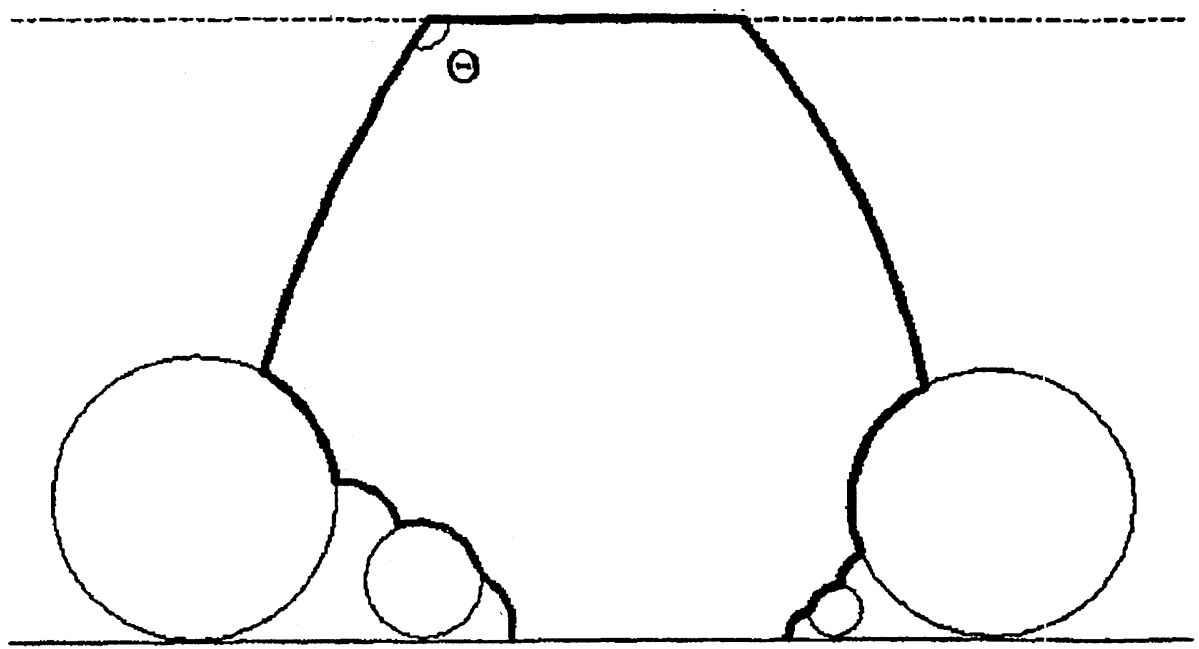

Figure 1: Broken curve $\zeta_{i}$ consisting of quasi-geodesic and quasi-cusp arcs.

There is a choice of injectivity radius $\epsilon_{\delta} \leq \epsilon_{2}$, so that the angle that the geodesic replacement arc of a quasi-cusp arc makes with the geodesic replacement of a quasi-geodesic arc is bounded above by $\pi-\delta$. One can visualize this by choosing $\epsilon_{\delta}$ sufficiently small so that $\Theta$ is close to $\pi / 2$; the point is that we want the geodesic to travel a large bounded distance beyond $\partial C^{[\epsilon, \infty)}(\Gamma)$ in any cusp, and then use the fact that the quasi-isometries 
are nearly isometric for all large index $i$. The geodesic replacement arc of the quasi-geodesic arc is nearly vertical with respect to any component of $\partial C^{(0, \epsilon]}(\Gamma)$, and the geodesic replacement arc to a quasi-cusp arc will be nearly vertical as well. The angle deflection bound follows immediately.

Note also that if $\Theta$ is sufficiently close to $\pi / 2$, then we are guaranteed that there will be at least one geodesic replacement arc of some quasi-geodesic $\operatorname{arc}$ in $\zeta_{i}$ that has non-trivial intersection with $N_{i}$ for all large index $i$. We choose the injectivity radius $\epsilon_{\delta}$ sufficiently small to ensure that this happens.

Let $N_{\infty}=C^{\left[\epsilon_{\delta}, \infty\right)}(\Gamma)$. We let $\left\{\widehat{\zeta}_{i}\right\}$ be the sequence of broken curves built from the sequence of pushforwards $\left\{\widehat{\beta}_{i} \subset M(\Gamma)\right\}$, so that the geodesic pieces come from the intersection of $\widehat{\beta}_{i} \cap C^{\left[\epsilon_{\delta}, \infty\right)}(\Gamma)$, and the cusp arcs consist of curves on $\partial C^{[\epsilon, \infty)}(\Gamma) \cap M^{(0, \epsilon]}(\Gamma)$ that are constructed from pieces having the property that $\widehat{\beta}_{i} \cap M^{\left(0, \epsilon_{\delta}\right)}(\Gamma) \neq \emptyset$. Once again, we can find a choice of closed geodesics $\left\{\beta_{i} \subset M\left(\Gamma_{i}\right)\right\}$, so that the associated sequence of push-forwards $\left\{\widehat{\beta}_{i} \subset M(\Gamma)\right\}$ has the property that eventually for all large index $i$, then $\widehat{\beta}_{i} \cap M^{\left(0, \epsilon_{\delta}\right)}(\Gamma) \neq \emptyset$. If not, we are can conclude using Lemma 5.4 that $\widehat{\beta}_{i}$ stays a bounded distance from $x_{i}$; the desired contradiction.

We finish the argument by using Theorem 5.2.2. Normalize the piecewise geodesic we have just constructed so that one of its endpoints lies on the hyperbolic plane $P=\left\{(x, y, t) \in \mathbf{H}^{3}: x^{2}+y^{2}+t^{2}=1\right\}$ and let $l_{t}$ be the hyperbolic line $(0,0, t): t>0$. By choosing $\delta$ small enough, we can appeal to Theorem 5.2.2 to demonstrate that $l_{t}$ projects under the group action $\Gamma_{i}$ to the closed geodesic $\beta_{i}$ homotopic to $\zeta_{i}$, and so that the minimum distance between $\zeta_{i}$ and $\beta_{i}$ is bounded by some constant for all large index $i$. Thus dist $\left(x_{i}, \beta_{i}\right)$ is not converging to infinity, a contradiction.

We now use the Main Theorem to resolve whether certain algebraically convergent sequences of representations are in fact converging strongly. Recall that a Fuchsian group is a Kleinian group that preserves a closed round disc $D \subset \widehat{\mathbf{C}}$. More generally, a quasi-Fuchsian group $\Gamma$ is a Kleinian group that is a quasi-conformal deformation of a Fuchsian group $G$ of the first kind (i.e. $L_{G}=\partial D$.) The study of quasi-Fuchsian groups was initiated in the foundational papers of Bers [4] and Maskit [31]. In the following, assume that the Fuchsian group $G$ is isomorphic to the fundamental group of a closed surface $S_{g}$ of genus $g$. Thus the quotient space $\left(\mathbf{H}^{3} \cup \Omega(G)\right) / G$ is topologically $S_{g} \times[0,1]$. If we fix a discrete faithful isomorphism of $\pi_{1}\left(S_{g}\right)$ into $P S L(2, \mathbf{C})$, then this isomorphism determines an ordered pair of points $(l, s)$ in the Teichmüller space $T_{g}$ of $S_{g}$. 
Let $\pi=\pi_{1}\left(S_{g}\right)$, and denote by $V(\pi)$ the set of discrete representations of $\pi$ into $P S L(2, \mathbf{C})$, where the identification is defined up to conjugation by Möbius transformations. Endow $V(\pi)$ with the algebraic topology, and note that $T_{g} \times T_{g}$ is identified with the open subset of $V(\pi)$ consisting of faithful representations of $\pi$ whose images are quasi-Fuchsian grou.ps. If we fix the first factor, then there is a holomorphic embedding of $T_{g}$ into $V(\pi)$. Such an embedding is called a Bers' slice [4]; we denote a Bers' slice by $T_{g}(l)$, where $l$ is the fixed conformal structure in the first factor.

The closure of a Bers' slice (denoted $\overline{T_{g}(l)}$ ) in $V(\pi)$ is compact; we call the closure the Bers' compactification. A cusp $[\Gamma] \in \partial \overline{T_{g}(l)}$ is an equivalence class of Kleinian groups where each marking in the class isomorphic to $G$, and so that there exists a hyperbolic element $g \in G$ such that the isomorphism takes $g$ to a parabolic element $\gamma \in \Gamma$. ([1],[16], see also [27]). Heuristically, one can view a cusp as being formed by contracting one or more homotopically non-trivial curves in the surface $D^{\circ} / G$; where $D^{\circ}$ is the interior of $D$. A maximal cusp is a boundary point of $\overline{T_{g}(l)}$ so that a maximal number (explicitly $3 g-3$ ) of disjoint, non-peripheral curves in $D^{\circ} / G$ have been contracted. A maximal cusp, i.e. a marking in the equivalence class, is geometrically finite as a Kleinian group [25]. Note that the non-fixed surface in the conformal boundary at infinity of a maximal cusp has been reduced to a finite collection of thrice punctured spheres. We will use maximal cusps in Example 5.8.

A $B$-group is a finitely generated Kleinian group $\Gamma$ that has a simply connected invariant component $\Delta_{0} \subset \Omega(\Gamma)$. In particular, quasi-Fuchsian groups are examples of B-groups. Bers [4] showed that the boundary of each compactified slice contains B-groups whose region of discontinuity consists of exactly one connected and simply connected component. Such a Kleinian group is called a degenerate group. Degenerate groups are knovin to be geometrically infinite [21].

A B-group $\Gamma$ is regular if it is neither quasi-Fuchsian nor degenerate, and satisfies a condition on the horocycles of its primary parabolic elements (see [1] page 212 for the full definition of regular B-group). A regular Bgroup is geometrically finite and resides on the boundary of some Bers' compactification ([1],[28]).

We can use the Main Theorem to show that degenerate groups do not converge strongly to regular B-groups on the boundary of a compactified Bers' slice. This result has content, since the set of degenerate groups is a dense $G_{\delta}$ set in the algebraic topology on the boundary of a Bers' compactification (see [4]). 
Corollary 5.5. Let $\varphi_{i}: G \rightarrow \Gamma_{i}$ be a sequence of degenerate groups converging algebraically to a regular B-group $\varphi_{\infty}: G \rightarrow \Gamma$ on the boundary of some Bers' compactification $\overline{T_{g}(l)}$. Then the convergence is not strong.

We assume familiarity with the concept of Hausdorff dimension [19]. Results in [6] show that the limit set of any geometrically infinite group has Hausdorff dimension 2. Sullivan [39] and Tukia [43] have shown the Hausdorff dimension of the limit set of a geometrically finite group is strictly less than 2. C. Bishop and P. Jones (Corollary 10.1 of [6]) cite the example of a sequence of degenerate groups converging to a regular B-group on the boundary of some Bers' compactification as demonstrating that Hausdorff dimension is not in general continuous in the algebraic topology. However, a long-standing conjecture is:

Conjecture 5.6. The Hausdorff dimension of the limit set is continuous with respect to strong convergence.

Corollary 5.5 is indirect evidence the conjecture is true. We refer the reader to Corollary 6.4 for a result related to this conjecture.

Often new parabolic subgroups are being formed in the limit group of a convergent sequence of Kleinian groups. Assume that the sequence $\left\{\Gamma_{i}=\right.$ $\left.\varphi_{i}(G)\right\}$ is converging strongly to a geometrically finite group $\Gamma=\varphi_{\infty}(G)$. The following proposition shows that, far enough out along the sequence, the number of cusps in $M\left(\Gamma_{i}\right)$ is bounded above by the number of cusps in $M(\Gamma)$.

Fix a basepoint $x_{\infty} \in M(\Gamma)$, and suppose $0 \in \mathbf{H}^{3}$ is fixed so that $0 \in \pi_{\infty}^{-1}\left(x_{\infty}\right)$. Let gen $G$ be a generating set of $G$ so $\varphi_{\infty}(g): g \in \operatorname{gen} G$ is a side-pairing of $P_{0}(\Gamma)$. Choose an injectivity radius $\epsilon>0$ sufficiently small, so that $M^{(0, \epsilon]}(\Gamma)$ consists solely of cusps. The collection of cusps of $M(\Gamma)$ will be given by $\left\{E_{\infty}^{k}\right\}_{k=1}^{k=s_{\infty}}$. Since $\Gamma$ is geometrically finite, it is clear that $s_{\infty}$ is finite (more generally, a finitely generated group has only a finite number of inequivalent parabolic subgroups, see [38]).

Proposition 5.7. Suppose that $\left\{\Gamma_{i}=\varphi_{i}(G)\right\}$ converges strongly to a geometrically finite group $\Gamma=\varphi_{\infty}(G)$, and assume that $M(\Gamma)$ has $s_{\infty} \geq 1$ cusps. Then for all index $i$ sufficiently large, $M\left(\Gamma_{i}\right)$ has $0 \leq s_{i} \leq s_{\infty}$ cusps.

We remark that the case $s_{\infty}=0$ (i.e. $\Gamma$ is convex co-compact) has been handled by the special case of the Main Theorem. 
Proof. Suppose not; assume there exists a subsequence $\left\{\Gamma_{j}\right\}_{j=1}^{j=\infty} \subseteq\left\{\Gamma_{i}\right\}$ so that $M\left(\Gamma_{j}\right)$ has $s_{j}>s_{\infty}$ cusps.

Let $\mathcal{P}=\left\{g_{1}, g_{2}, \ldots, g_{\eta}\right\}\left(\eta \leq 2 s_{\infty}\right)$ be the sub-collection of gen $G$ so that $\left\{\varphi_{\infty}\left(g_{n}\right)\right\}$ generate the subgroups in $\Gamma$ that uniformize $\left\{E_{\infty}^{k}\right\}_{k=1}^{k=s_{\infty}}$. Since $\left\{\varphi_{j}\right\}$ is converging strongly to $\varphi_{\infty}$, observe that $\varphi_{j}\left(g_{n}\right) \rightarrow \varphi_{\infty}\left(g_{n}\right)$, where $1 \leq n \leq \eta$. Thus there is a sequence of convex sets $\left\{E_{j}^{k} \subset M\left(\Gamma_{j}\right), 0 \leq\right.$ $\left.k \leq s_{\infty}\right\}$, so that each $E_{j}^{k}$ is converging to $E_{\infty}^{k}$. We denote by $E_{j}^{s_{\infty}+1}$ a component in $\left\{E_{j}^{k}\right\}_{k=s_{\infty}+1}^{k=s_{j}}$. Let $G(\mathcal{P}) \subset G$ be the collection of elements in $G$ so that $\varphi_{\infty}(g): g \in G(\mathcal{P})$ is parabolic in $\Gamma$. Note that because $\Gamma$ is non-elementary, then $G-G(\mathcal{P}) \neq \emptyset$.

We can find, using a diagonalization argument if necessary, a sequence of closed geodesics $\left\{\beta_{j} \subset M\left(\Gamma_{j}\right)\right\}$ so that

$$
\operatorname{Dist}\left(x_{j}, \beta_{j}\right) \rightarrow \infty
$$

as $j \rightarrow \infty$; as before $x_{j}=\pi_{j}(0)$. (Recall Dist $\left(x_{j}, \beta_{j}\right)=\max \left\{\rho\left(x_{j}, y\right): y \in\right.$ $\left.\beta_{j}\right\}$.)

We further specify the homotopy type of each $\beta_{j}$. Recall a closed geodesic has the form $\beta=A / \gamma$, where $A$ is the axis of some loxodromic element $\gamma$. Choose $\gamma_{j}=\Pi_{k=1}^{k=k(j)} \varphi_{j}\left(g_{k}\right)$ so $g_{k} \in$ gen $G-G(\mathcal{P})$, and let $\beta_{j}=A_{j} / \gamma_{j}$. We explicitly form the sequence by finding a collection of geodesics, each of which has the property that for large index $j$

$$
\beta_{j} \cap M^{(0, \epsilon / 2]}\left(\Gamma_{j}\right) \subset E_{j}^{s_{\infty}+1} .
$$

One readily sees that such a choice of geodesics can be found: Recall that by assumption there is at least one cusp $E_{j}^{s_{\infty}+1}$ that is not in the set that is converging to the collection $\left\{E_{\infty}^{k}\right\}$.

Let $N_{\infty}=\mathcal{N}_{1} C^{[\epsilon, \infty)}(\Gamma)$, by our choice of injectivity radius $\epsilon$ it is a compact core of $M(\Gamma)$. By Proposition 5.1 , we observe that $N_{j}=f_{j}^{-1}\left(N_{\infty}\right)$ is a compact core of $M\left(\Gamma_{j}\right)$ for all index $j$ sufficiently large.

For all such index $j$, let $\alpha_{j} \subset N_{j}$ be a curve homotopic to $\beta_{j}$. Map $\alpha_{j}$ to $\widehat{\alpha}_{j}=f_{j}\left(\alpha_{j}\right)$ in $N_{\infty}$. The curve $\widehat{\alpha}_{j}$ is homotopically non-trivial in $\pi_{1}(M(\Gamma))$, so there is a geodesic $\widehat{\beta}_{j} \subset M\left(\Gamma_{j}\right)$ homotopic to $\widehat{\alpha}_{j}$. Since this geodesic travels at most a bounded distance in any of the $E_{\infty}^{k}$ (else it picks up homotopy from the cusp), we conclude $\widehat{\beta}_{j}$ is properly contained in $N_{\infty}$. Now apply Lemma 5.4 , and observe that eventually $\beta_{j}$ is contained in $N_{j}$. As this contradicts (4), we are done. 
Example 5.8. We give a collection of examples showing that the statement and conclusion of Proposition 5.7 can not be weakened.

The following shows that the conclusion of Proposition 5.7 is not true if the limit is geometrically infinite. Let $\Gamma=\varphi_{\infty}(G)$ be a degenerate group without parabolics on the boundary of a Bers' slice $T_{g}$. By a result of McMullen [33], there exists a sequence of maximal cusps $\varphi_{i} \in \partial \overline{T_{g}}$ converging in the algebraic topology to $\varphi_{\infty}$. Using the covering theorem of Thurston (see Canary [12] and [42]), we observe that $\left\{\Gamma_{i}=\varphi_{i}(G)\right\}$ is in fact converging strongly to $\Gamma=\varphi_{\infty}(G)$. Note that $s_{i}=3 g-3$ for all index $i$, but $s_{\infty}=0$.

The assumption of algebraic convergence alone is not enough to assert the conclusion of the proposition. Fix a regular B-group $\Gamma$ in $\partial \overline{T_{g}}$, so that there is only one equivalence class of parabolics in $\Gamma$. In particular $\Gamma$ is geometrically finite. Since maximal cusps are dense, we can find a sequence of these that converge to $\Gamma$ in the algebraic topology. One shows this sequence is not converging strongly by implementing the argument in the proof of Proposition 5.7.

We construct an example of a geometrically convergent sequence, with no algebraic limit, so that $\liminf s_{i}>s_{\infty}$. Fix two disjoint round closed discs $D_{i=1,2} \subset\{z \in \mathbf{C}:$ re $z<0\}$, and let $\gamma$ be a loxodromic element that takes the exterior of $D_{2}$ to the interior of $D_{1}$. Likewise, fix two round closed discs $D_{i=3,4} \subset\{z \in \mathbf{C}: r e z>0\}$, so $\{c\}=D_{3} \cap D_{4}$ (necessarily $r e c>0$ ). Let $p$ be a parabolic Möbius transformation that fixes $c$ and takes the exterior of $D_{3}$ to the interior of $D_{4}$. By an application of the Klein Combination Theorem (Theorem VII.A.13 in [32]) the group $\Gamma$ generated by $G_{1}=<\gamma>$ and $G_{2}=<$ $p>$ is discrete, and $F=\left(\widehat{\mathbf{C}}-\left(D_{1} \cup D_{2}\right)\right) \cap\left(\widehat{\mathbf{C}}-\left(D_{3} \cup D_{4}\right)\right)$ is a fundamental domain for $\Gamma$. In particular, $\Gamma$ contains one conjugacy class of parabolics and $\infty \in \Omega(\Gamma)$. Let $\left.G_{2, i}=<\omega^{i} \circ p \circ \omega^{-i}\right\rangle$, where $\omega^{i}(z)=z+i$ for $i \in \mathbf{Z}^{+}$. For each index $i$, an application of the Klein Combination Theorem implies that $\Gamma_{i}=<G_{1}, G_{2, i}>$ is discrete and $F_{i}=\left(\widehat{\mathbf{C}}-\left(D_{1} \cup D_{2}\right)\right) \cap\left(\widehat{\mathbf{C}}-\omega^{i}\left(D_{3} \cup D_{4}\right)\right)$ is a fundamental domain for $\Gamma_{i}$. By construction $\Gamma_{i}$ contains one conjugacy class of parabolic elements. We form the sequence $\left\{\Gamma_{i}\right\}$. One observes that $\left\{\Gamma_{i}\right\}$ is converging geometrically to $G_{1}$ (see the definition of polyhedral convergence, and its equivalence to geometric convergence, given in the next section.) The sequence is not converging algebraically.

\section{Geometric Convergence and Convex Co-compact Limits.}

We assume familiarity with the definition of the Hausdorff set metric ([19],[24]). 
The main result of this section is:

Theorem 6.1. Let $\left\{\Gamma_{i}\right\}$ be a sequence of Kleinian groups converging geometrically to a convex co-compact limit $\Gamma$. Further, assume the associated sequence of limit sets $\left\{L_{\Gamma_{i}}\right\}$ is converging in the Hausdorff set metric to the limit set $L_{\Gamma}$. Then there is an index $\mathcal{I}$ so that for each $i>\mathcal{I}$ the group $\Gamma_{i}$ is convex co-compact.

Proof. Since $\Gamma$ is convex co-compact, the 1-neighborhood of the convex core is a compact and the manifold $M(\Gamma)-\mathcal{N}_{1} C(\Gamma)$ is homeomorphic to $\partial \mathcal{N}{ }_{1} C(\Gamma) \times$ $[0, \infty)([34],[42])$. Fix a point $x_{\infty} \in M(\Gamma)$, and let $0 \in \pi_{\infty}^{-1}\left(x_{\infty}\right)$. Form the Dirichlet polyhedron $P_{0}(\Gamma)$, and let $A_{\infty}=P_{0}(\Gamma) \cap \pi_{\infty}^{-1}(C(\Gamma))$. We also fix, for now, a radial length $\tilde{r}$ sufficiently large so that $\mathcal{N}_{1} C(\Gamma) \subset \pi_{\infty}(B(0, \tilde{r}))$.

Assume that the index $i$ is large enough so that $f_{i}\left(V_{i}\right) \supset \mathcal{N}_{1}(C(\Gamma)$ ) (see Theorem 3.1 (3)), and consider the submanifolds $C_{i}=f_{i}^{-1}\left(\mathcal{N}_{1} C(\Gamma)\right)$. We claim that eventually each $C_{i}$ is a compact core of $M\left(\Gamma_{i}\right)$.

For suppose not. Then for all large index $i$, there exists homotopically non-trivial curve $\alpha^{i} \in \pi_{1}\left(M\left(\Gamma_{i}\right)\right)$ so that the (unique) geodesic $\beta_{i}$ in the homotopy class $\left[\alpha^{i}\right]$ has non-trivial intersection with $M\left(\Gamma_{i}\right)-\pi_{i}(B(0, \tilde{r}))$. If no such geodesic $\beta_{i}$ existed, then clearly for all large index $i$ the submanifold $\pi_{i}(B(0, \tilde{r}))$ would contain all of the closed geodesics in $M\left(\Gamma_{i}\right)$, and as such is a compact core of $M\left(\Gamma_{i}\right)$ as desired.

Now we claim that the existence of such a close geodesic $\beta_{i}$ is enough to show that $\left\{L_{\Gamma_{i}}\right\}$ is not converging to $L_{\Gamma}$. Because $\beta_{i} \cap\left(M\left(\Gamma_{i}\right)-\right.$ $\left.\pi_{i}(B(0, \tilde{r}))\right) \neq \emptyset$, there is a lift of $\beta_{i}$ that has non-trivial intersection with $P_{0}(\Gamma)-B(0, \tilde{r})$. By making the radial length $\tilde{r}$ larger, if necessary, one (or both) of the endpoints (i.e. loxodromic fixed points in $L_{\Gamma_{i}}$ ) of this lift lie in $\overline{P_{0}(\Gamma)} \cap \widehat{\mathbf{C}}$; here $\overline{P_{0}(\Gamma)}$ is the Euclidean closure of $P_{0}(\Gamma)$. (If we must increase the value of $\tilde{r}$ then we might have to amend our choice of the closed geodesic $\beta_{i}$. However, regardless of how large the fixed value of $\hat{r}$ is, such a $\beta_{i}$ must exist, else we can assert that all of the closed geodesics in $M\left(\Gamma_{i}\right)$ are contained in $\pi_{i}(B(0, \tilde{r}))$.) Since $\Gamma$ is convex co-compact the closed set $\overline{P_{0}(\Gamma)} \cap \widehat{\mathbf{C}}$ is properly contained in $\Omega(\Gamma)$. Thus at least one of the losiodromic fixed points in $L_{\Gamma_{i}}$ associated to the closed geodesic $\beta_{i}$ remains a bounded distance away from $L_{\Gamma}$ for all large index $i$. This contradicts the assumption of limit set convergence; explicitly, for large index $i$, each $C_{i}$ is a compact core of $M\left(\Gamma_{i}\right)$ and so $\Gamma_{i}$ is isomorphic to $\Gamma$.

We now show that $C_{i}$ contains the convex core of $M\left(\Gamma_{i}\right)$. Recall that $\left\{\mathrm{E}_{\Gamma_{i}}\right\}$ is assumed to be converging in the Hausdorff set metric to $L_{\Gamma}$; thus 
$C H\left(L_{\Gamma_{i}}\right) \cap B(0, \tilde{r})$ is converging to $C H\left(L_{\Gamma}\right) \cap B(0, \tilde{r})$ (see [9]) in the Hausdorff set metric in $\mathbf{H}^{3}$. Let $A_{i}=C H\left(L_{\Gamma_{i}}\right) \cap B(0, \tilde{r})$, then $\pi_{i}\left(A_{i}\right) \subset C\left(\Gamma_{i}\right)$. Since the $C_{i}$ are compact cores each $\left[\gamma_{i}\right] \in \pi_{1}\left(M\left(\Gamma_{i}\right)\right)-[1]$ has a representative in $C_{i}$. But each such curve in $C_{i}$ is a $k_{i}$-quasi-isometric image of a closed geodesic, hence by Lemma 5.4 we observe that all of the closed geodesics in $M\left(\Gamma_{i}\right)$ lie within a bounded distance of $f_{i}^{-1}(C(\Gamma)) \subset C_{i}$. Since the $f_{i}$ are diffeomorphisms, we can conclude that all of the closed geodesics in $M\left(\Gamma_{i}\right)$ are in $C_{i}$. Thus via the observation at the beginning of the Main Theorem (special case), we can conclude $\pi_{i}\left(A_{i}\right) \supset C\left(\Gamma_{i}\right)$. Therefore $\pi_{i}\left(A_{i}\right)=C\left(\Gamma_{i}\right)$, and because $\pi_{i}\left(A_{i}\right)$ is compact (as the continuous image of a compact set), we are done.

Remark: It is not true that a sequence $\left\{\Gamma_{i}\right\}$ of Kleinian groups converging geometrically to a geometrically finite group $\Gamma$ with cusps, so that the limit sets converge, implies that eventually the $\Gamma_{i}$ is geometrically finite for all large index $i$. Example 6.2 illustrates this phenomena. The basic problem in the presence of parabolics is that the assumptions of limit set convergence and geometric convergence does not guarantee that the $C_{i}$ (as defined in the argument above) are eventually compact cores. Non-trivial topology might be "pushed out"the cusp, and thus disappear in the limit while still having the limit sets converge.

The following example shows it is possible to have a sequence of geometrically infinite groups converging geometrically to a geometrically finite limit group with parabolics, so the limit sets converge as well.

We start with the definition of polyhedral convergence. A sequence of discrete groups $\left\{\Gamma_{i}\right\}$ converges polyhedrally (see [24]) to a group $\Gamma$ if $\Gamma$ is discrete, and for a fixed $0 \in \mathbf{H}^{3}$ the sequence of Dirichlet polyhedra $\left\{P_{0}\left(\Gamma_{i}\right)\right\}$ converge uniformly to $P_{0}(\Gamma)$ on compact subsets of $\mathbf{H}^{3}$. We express this more concretely: Fix a radial length $\tilde{r}>0$ and let $B(0, \tilde{r})$ be the closed ball about 0 of radius $\tilde{r}$. Polyhedral convergence means there exists an index $N(\tilde{r})$ so that

1. for each face pairing transformation of $P_{0}(\Gamma) \cap B(0, \tilde{r})$ there exists for all $i>N(\tilde{r})$ a face pairing transformation $\gamma_{i}$ of $P_{0}\left(\Gamma_{i}\right) \cap B(0, \tilde{r})$ so that $\lim \gamma_{i}=\gamma$, and

2. if $\gamma_{i} \in \Gamma_{i}$ is a face-pairing transformation of $P_{0}\left(\Gamma_{i}\right) \cap B(0, \tilde{r})$, then the limit of any convergent subsequence of $\left\{\gamma_{i}\right\}$ is a face, edge or vertex pairing transformation of $P_{0}(\Gamma) \cap B(0, \tilde{r})$. 
Jørgensen and Marden [24] showed that a sequence $\left\{\Gamma_{i}\right\}$ of Kleinian groups converges geometrically to a Kleinian group $\Gamma$ if and only if $\left\{\Gamma_{i}\right\}$ converges polyhedrally to $\Gamma$.

Our construction of this example makes use of the polyhedral clefinition of geometric convergence; we realize a rank 1-cusp in the geometrically finite limit manifold as the polyhedral limit of a sequence of geometrically infinite ends.

We show limit set convergence in Example 6.2 by demonstrating that the regular sets are converging in a certain way. Let $\left\{O_{i}\right\}$ be a secuence of open sets in $\widehat{\mathbf{C}}$. We say that $\left\{O_{i}\right\}$ is converging in the sense of Carcitheodory to an open set $O$ if

1. every compact set $K \subset O$ lies in $O_{i}$ for all index $i$ sufficiently large, and

2. every open set $U$ in $O_{j}$ for infinitely many index $j$ also lies in $O$.

One can easily show that Caratheodory convergence of the sequence of regular sets $\left\{\Omega\left(\Gamma_{i}\right)\right\}$ to $\Omega(\Gamma)$ is equivalent to Hausdorff convergence of the sequence of limit sets $\left\{L_{\Gamma_{i}}\right\}$ to $L_{\Gamma}$ (see [24]).

Because we will be concerned with neighborhoods of the point at infinity on $\widehat{\mathbf{C}}$ in the following example, there is a need to introduce a special metric. The chordal metric is the metric on $\widehat{\mathbf{C}}$ induced by the stereographic projection of $\widehat{\mathbf{C}}$ onto $S^{2} \subset \mathbf{R}^{3}$. In effect, the Euclidean metric on $S^{2} \subset \mathbf{R}^{3}$ is transferred to a metric on $\widehat{\mathbf{C}}$. (See [2] for a more detailed exposition concerning the chordal metric.)

Example 6.2. Let $\widehat{\Gamma}$ be a degenerate group ([4],[31]) with a parabolic subgroup; recall that $\widehat{\Gamma}$ is geometrically infinite [21]. Conjugate $\widehat{\Gamma}$ so that this parabolic subgroup is generated by $p(z)=z+1$. We can also conjugate $\widehat{\Gamma}$ with a sufficiently high power of $m(z)=z-i$ so that $U=\{z \in \widehat{\mathbf{C}}: i m z>0\}$ is precisely invariant under $\Gamma^{\prime}-\left\langle p(z)>\left(\right.\right.$ here $\left.\Gamma^{\prime}=m^{k} \circ \widehat{\Gamma} \circ m^{-k}\right)$. The strategy is to build a geometrically finite group $\Gamma$ containing $\langle z \rightarrow z+1\rangle$ so we can form an amalgamated free product of $\Gamma^{\prime}$ with $\Gamma$ across $\langle z \rightarrow z+1\rangle$. We then push the geometrically infinite factor towards the parabolic fixed point $\infty \in \widehat{\mathbf{C}}$.

Let $\Gamma_{1}$ be a loxodromic cyclic group and let $\Gamma_{2}$ be $\langle p(z)\rangle$. Choose the generator $g(z)$ of $\Gamma_{1}$ so that $\Gamma=<\Gamma_{1}, \Gamma_{2}>$ is discrete and a free product of $\Gamma_{1}$ and $\Gamma_{2}$. We also impose as a condition on the choice of $g^{\prime}(z)$ that $L=\{z \in \widehat{\mathbf{C}}: i m z<0\}$ be precisely invariant under $\Gamma-\Gamma_{2}$ (choose $g(z)$ to be a loxodromic element so that the centers of its isometric circles are on the 
positive imaginary axis and the radii of the isometric circles are sufficiently small). $\Gamma$ is a generalized Schottky group of rank 2, and it is geometrically finite by construction. Observe that $(U, L)$ is a proper interactive pair, and $\bar{U}$ is a $\left(<p(z)>, \Gamma^{\prime}\right)$-block and $\bar{L}$ is a $(<p(z)>, \Gamma)$-block (see [32] pages 136-141 for the definition of proper interactive pair and block).

Thus we can use the Maskit Combination Theorem I (Theorem VII.C.2 in [32]) to form the amalgamated free product

$$
\tilde{\Gamma}=\Gamma *<p(z)>\Gamma^{\prime} .
$$

$\tilde{\Gamma}$ is geometrically infinite ([32] VII.C.2.xi).

Now push $\Gamma^{\prime}$ to infinity along the cusp. To do so, let

$$
\Gamma_{i}^{\prime}=m^{i} \circ \Gamma^{\prime} \circ m^{-i}
$$

where $m(z)$ is as above and form

$$
\tilde{\Gamma}_{i}=\Gamma *<p(z)>\Gamma_{i}^{\prime} .
$$

We can apply the Combination Theorem I because by construction $(U, L)$ a proper interactive pair, and $\bar{U}$ is a $\left(<p(z)>, \Gamma_{i}^{\prime}\right)$-block and $\bar{L}$ is a $(<$ $p(z)>, \Gamma)$-block. Fix a basepoint $z=(x, i y, t) \in \mathbf{H}^{3}$. For any $r>0$ then $P_{z}\left(\tilde{\Gamma}_{i}\right)$ converges uniformly to $P_{z}(\Gamma)$ on $B(z, r) \subset \mathbf{H}^{3}$. From the equivalence of polyhedral convergence and geometric convergence we deduce that $\left\{\tilde{\Gamma}_{i}\right\}$ converges geometrically to $\Gamma$.

It remains to show $\left\{L_{\Gamma_{i}}\right\}$ converges to $L_{\Gamma}$ in the Hausdorff set metric on C. Equivalently, we demonstrate that the sequence of regular sets $\left\{\Omega\left(\tilde{\Gamma}_{i}\right)\right\}$ is converging in the sense of Caratheodory to $\Omega(\Gamma)$. Let $F_{i}^{\prime}$ and $F_{\Gamma}$ be the fundamental sets formed by the intersection of the Euclidean closure of $P_{z}\left(\Gamma_{i}^{\prime}\right)$ and $P_{z}(\Gamma)$ respectively with $\widehat{\mathbf{C}}$. One concludes from statement VII.C.2.vii in [32] that

$$
\tilde{F}_{i}=\left(F_{i}^{\prime} \cap \bar{L}\right) \cup\left(F_{\Gamma} \cap \bar{U}\right)
$$

is a fundamental set for $\tilde{\Gamma}_{i}$. Let $K$ be a compact set in $\Omega(\Gamma) ; K$ is a bounded distance (in the chordal metric) from $L_{\Gamma}$. Note that $\infty \in L_{\Gamma}$ and $F_{i}^{\prime} \cap L$ is being contracted in the chordal metric about the point $\infty$ as $i \rightarrow \infty$. Thus we observe that eventually $K \subset \Omega\left(\tilde{\Gamma}_{i}\right)$. Caratheodory convergence of the regular sets $\left\{\Omega\left(\tilde{\Gamma}_{i}\right)\right\}$ to $\Omega(\Gamma)$ follows immediately.

Remark: Jørgensen and Marden [24] showed that a sequence of discrete faithful representations converging algebraically to a geometrically finite infinite volume representation $\varphi_{\infty}$, so that the limit sets are converging, implies that the sequence of groups $\Gamma_{i}=\varphi_{i}(G)$ is converging geometrically to 
$\Gamma=\varphi_{\infty}(G)$ as well. In Example 6.2, since the geometric limit $\Gamma^{\prime}$ is properly contained in $\tilde{\Gamma}_{i}$ for all index $i$, we observe that $\left\{\tilde{\Gamma}_{i}\right\}$ is not converging strongly to $\Gamma$. Thus the Jørgensen-Marden result is the best possible: One can not assume geometric convergence rather than algebraic convergence in the statement of their theorem. This observation, using a different class of examples, was known to both Jørgensen and Marden [29].

We have one more application for polyhedral convergence. By analyzing the proof of Theorem 6.1 in more depth, and using the polyhedral formulation of geometric convergence, we can actually prove a stronger result. The reader is referred to [5] for the definition of quasi-conformal conjugacy.

Lemma 6.3. Suppose that $\left\{\Gamma_{i}\right\}$ converges geometrically to a convex cocompact group $\Gamma$ and assume that $\left\{L_{\Gamma_{i}}\right\}$ converges in the Hausdorff set metric to $L_{\Gamma}$. Then $\Gamma_{i}$ is $K_{i}$-quasi-conformally conjugate to $\Gamma$ for all large index i. In fact, $K_{i} \rightarrow 1$ as $i \rightarrow \infty$.

Proof. Fix $0 \in \mathbf{H}^{3}$. We have already shown that, under these hypotheses, $\Gamma_{i}$ is eventually convex co-compact. It was also shown that for a radial length $\tilde{r}$ large enough so $\mathcal{N}_{1} C(\Gamma) \subset B(0, \tilde{r}) / \Gamma$, then eventually $C\left(\Gamma_{i}\right) \subset E^{\prime}(0, \tilde{r}) / \Gamma_{i}$. Let $N_{\infty}=\mathcal{N}_{1} C(\Gamma)$ and $N_{i}=f_{i}^{-1}\left(N_{\infty}\right)$.

Since $\Gamma_{i}$ is eventually convex co-compact, and $\Gamma$ is assumed to be convex co-compact, then $C\left(\Gamma_{i}\right)$ is a compact core of $M\left(\Gamma_{i}\right)$ and likewise $C(\Gamma)$ is a compact core of $M(\Gamma)$. Thus for all index $i$ large enough so that $C\left(\Gamma_{i}\right) \subset$ $f_{i}^{-1}\left(\mathcal{N}_{1} C(\Gamma)\right)$, the diffeomorphism $f_{i}$ induces an isomorphism of $\Gamma_{i}$ to $\Gamma$.

Now consider the polyhedral convergence of $\left\{P_{0}\left(\Gamma_{i}\right)\right\}$ to $P_{0}(\Gamma)$ on $B(0, \tilde{r})$, induced by the geometric convergence of the groups. Recall that the sidepairings a fundamental polyhedron generate the group ([32] IV.F.6.). Since $\Gamma_{i}$ is eventually isomorphic to $\Gamma$, then the full set of side-pairings of $P_{0}\left(\Gamma_{i}\right)$ are converging to the full set of side-pairings of $P_{0}(\Gamma)$ uniformly on $B(0, \tilde{r})$.

Fix $\epsilon>0$, and let $\left\{\gamma_{j}\right\}_{j=1}^{j=\kappa}$ be the (finite) collection of generators of $\Gamma$. Then we have shown that eventually $\left|\gamma-\varphi_{i}(\gamma)\right|<\epsilon$, where $|\cdot|$ is the standard norm on $\operatorname{PSL}(2, \mathbf{C})$. We now apply Marden's Stability Theorem (Proposition 9.1 in [27]) to finish the proof of the lemma. The fact that $K_{i} \rightarrow 1$ follows by making $\epsilon$ arbitrarily small.

We return briefly to the question of continuity of Hausdorff climension (see Conjecture 5.6). Using estimates on the distortion of Hausdorff dimension by $K$-quasi-conformal mappings in Gehring-Väisälä [20], we have 
the following immediate corollary of Lemma 6.3. We denote the Hausdorff dimension of a set $A \subseteq \widehat{\mathrm{C}}$ by $d(A)$.

Corollary 6.4. Suppose that $\left\{\Gamma_{i}\right\}$ converges geometrically to a convex cocompact group $\Gamma$ and assume $\left\{L_{\Gamma_{i}}\right\}$ converges in the Hausdorff set metric to $L_{\Gamma}$. Then $\left\{d\left(L_{\Gamma_{i}}\right)\right\}$ converges to $d\left(L_{\Gamma}\right)$.

\section{Volumes of Convex Cores.}

It will be shown that the volume function, restricted to the convex core, is continuous on a strongly convergent sequence of Kleinian groups. We start by showing that the volume function restricted to the convex core is lower semi-continuous under the assumption of geometric convergence. The following lemma is true for an arbitrary sequence of geometrically convergent Kleinian groups with a non-elementary limit; we need not make any assumptions about the groups being geometrically finite or torsion-free. A stronger version of Lemma 7.1 is proved in [26] (Proposition 2.1).

Lemma 7.1. Let $\left\{\Gamma_{i}\right\}$ be a sequence of Kleinian groups converging geometrically to a non-elementary group $\Gamma$. Then there exists a convergent subsequence of $\left\{L_{\Gamma_{i}}\right\}$, and a set $\Lambda \subseteq \widehat{\mathbf{C}}$, so that $\Lambda$ is the limit in the Hausdorff set metric of the subsequence. Each such set $\Lambda$ is closed and $\Gamma$-invariant; in particular $L_{\Gamma} \subseteq \Lambda$.

Proof. Because of the assumption that the geometric limit is non-elementary we know that each $\Gamma_{i}$ is non-elementary for all large index $i$. Recall the definition and properties of the Hausdorff topology on the family $C(\widehat{\mathbf{C}})$ of closed subsets of the Riemann sphere. Because $\widehat{\mathbf{C}}$ is locally compact there is a convergent subsequence $\left\{L_{\Gamma_{j}}\right\} \subseteq\left\{L_{\Gamma_{i}}\right\}$ with limit $\Lambda$. The set $\Lambda$ is necessarily closed.

We claim that $\Lambda$ is non-empty. Let $x_{j} \in L_{\Gamma_{j}}$; by the compactness of $\widehat{\mathbf{C}}$ there is a convergent subsequence $\left\{x_{k}\right\} \subseteq\left\{x_{j}\right\}$ with limit $x_{\infty} \in \widehat{\mathbf{C}}$. Since $\Lambda$ is the limit of $\left\{L_{\Gamma_{j}}\right\}$ in the Hausdorff topology, we observe that $x_{\infty} \in \Lambda$.

It remains to show that $\Lambda$ is $\Gamma$-invariant. Let $\gamma \in \Gamma$, then by geometric convergence of the groups there exists a sequence $\gamma_{j} \in \Gamma_{j}$ so $\gamma_{j} \rightarrow \gamma$ in the compact-open topology. Let $x_{j} \in L_{\Gamma_{j}}$ be converging to $x \in \Lambda$. Then for $j$ sufficiently large $\gamma_{j}\left(x_{j}\right) \rightarrow \gamma(x)$. But $\gamma_{j}\left(x_{j}\right) \in L_{\Gamma_{j}}$ because $L_{\Gamma_{j}}$ is $\Gamma_{j}$-invariant. Hence $\gamma(x) \in \Lambda$. 
Since the limit set of a non-elementary group is the smallest closed $\Gamma$ invariant subset of the Riemann sphere, then any closed $\Gamma$-invariant set $\Lambda$ contains the limit set $L_{\Gamma}$.

Let $C_{\Lambda}(\Gamma)=C H(\Lambda) / \Gamma$; because $L_{\Gamma} \subseteq \Lambda$ it is clear that $C(\Gamma) \subseteq C_{\Lambda}(\Gamma)$. The strategy in the proof of Theorem 7.2 is to show lower semi-continuity by pulling back large pieces of $C_{\Lambda}(\Gamma)$ to $M\left(\Gamma_{i}\right)$ via the quasi-isometries induced by the geometric convergence. We then use the fact that $L_{\Gamma_{j}} \rightarrow \Lambda$.

Theorem 7.2. Suppose $\left\{\Gamma_{i}\right\}$ converges geometrically to $\Gamma$, then

$$
\liminf _{i \rightarrow \infty} \operatorname{vol} C\left(\Gamma_{i}\right) \geq \operatorname{vol} C(\Gamma) .
$$

If vol $C(\Gamma)=0$ the inequality is trivially satisfied, so assume that $\Gamma$ is a non-elementary Kleinian group whose limit set does not lie on a round circle. Let $\Lambda$ and $\left\{L_{\Gamma_{j}}\right\}$ be as in Lemma 7.1, and fix points $z \in C_{\Lambda}(\Gamma)$ and $0 \in \pi^{-1}(z)$. Truncate $C_{\Lambda}(\Gamma)$ with a closed ball of radius $r$ about $z$, i.e. let $C_{\Lambda}^{r}(\Gamma)=C_{\Lambda}(\Gamma) \cap B(z, r)$. Note that $\lim _{r \rightarrow \infty} \operatorname{vol} C_{\Lambda}^{r}(\Gamma)=\operatorname{vol} C_{\Lambda}(\Gamma)$.

Using a result of Bowditch [9], we observe that $\left\{\partial C H\left(L_{\Gamma_{j}}\right) \cap B(0, r)\right\}$ is converging in $\mathbf{H}^{3}$ to $\partial C H(\Lambda) \cap B(0, r)$ in the Hausdorff set metric. Recall (Theorem 3.1) that the maps $\left\{f_{j}\right\}$ are $k_{j}$-quasi-isometries with $k_{j} \rightarrow 1$. It is an easy observation that for any $\delta>0$ there is an index $N$ so that for all $j>N$ then $f_{j}^{-1}\left(C_{\Lambda}^{r}(\Gamma)\right) \subset \mathcal{N}_{\delta}\left(C^{r}\left(\Gamma_{j}\right)\right)$ and $f_{j}\left(C^{r}\left(\Gamma_{j}\right)\right) \subset \mathcal{N}_{\delta}\left(C_{\Lambda}^{r}(\Gamma)\right)$. We can immediately conclude that

$$
\lim \operatorname{vol} C^{r}\left(\Gamma_{j}\right)=\operatorname{vol} C_{\Lambda}^{r}(\Gamma) .
$$

We now consider the two possible cases: vol $C(\Gamma)=\infty$ and vol $C(\Gamma)$ is bounded. Suppose vol $C(\Gamma)=\infty$, then because $\Lambda \supseteq L_{\Gamma}$ we have vol $C_{\Lambda}(\Gamma)=$ $\infty$. Thus for any large $V$ there exists a radial length $r$ so that vol $C_{\Lambda}^{r}(\Gamma)>2 V$. Hence there exists an index $N$ so that for all $j>N$ then vol $C^{r}\left(\Gamma_{j}\right)>V$. Since vol $C\left(\Gamma_{j}\right) \geq \operatorname{vol} C^{r}\left(\Gamma_{j}\right)$, we observe that lim vol $C\left(\Gamma_{j}\right)=\infty$.

Now suppose that $\liminf _{i \rightarrow \infty}$ vol $C\left(\Gamma_{i}\right)<\infty$. There exists a subsequence $\left\{\Gamma_{k}\right\} \subset\left\{\Gamma_{i}\right\}$ such that vol $C\left(\Gamma_{k}\right)<L<\infty$ for all $k$. By compactness there exists a subsequence (again denoted by $\left\{\Gamma_{k}\right\}$ ) such that $\left\{\Gamma_{k}\right\}$ converges geometrically to $\Gamma$. Apply Lemma 7.1 to find yet another subsequence $\left\{\Gamma_{s}\right\} \subseteq\left\{\Gamma_{k}\right\}$ such that $\left\{L_{\Gamma_{s}}\right\}$ converges in the Hausdorff set metric to a closed set $\widehat{\Lambda} \supseteq L_{\Gamma}$. The analysis above implies that $\liminf _{s \rightarrow \infty} \operatorname{vol} C\left(\Gamma_{s}\right)=\infty$, a contradiction. 
Suppose that vol $C_{\Lambda}(\Gamma)<\infty$ (if vol $C_{\Lambda}(\Gamma)=\infty$ we are done by the argument given above.) Fix $\epsilon>0$, and let $r$ be a radial length sufficiently large so that vol $C_{\Lambda}^{r}(\Gamma)>$ vol $C_{\Lambda}(\Gamma)-\epsilon$. Since $\lim$ vol $C^{r}\left(\Gamma_{j}\right)=\operatorname{vol} C_{\Lambda}^{r}(\Gamma)$, we observe that eventually vol $C^{r}\left(\Gamma_{j}\right)>$ vol $C_{\Lambda}(\Gamma)-\epsilon$. Thus since $L_{\Gamma} \subseteq \Lambda$ then

$$
\liminf _{j \rightarrow \infty} \operatorname{vol} C\left(\Gamma_{j}\right) \geq \operatorname{vol} C(\Gamma) .
$$

Now apply a similar analysis as in the vol $C(\Gamma)=\infty$ case.

As an immediate consequence of this theorem, if the sequence $\left\{\varphi_{i}: \varphi_{i}\right.$ : $\left.G \rightarrow \Gamma_{i}\right\}$ is converging strongly to a geometrically infinite limit representation $\varphi_{\infty}$, then the sequence of volumes $\left\{\operatorname{vol} C\left(\Gamma_{i}\right)\right\}$ is converging to infinity. Thus we can reduce the problem of showing volume continuity to the consideration of a strongly convergent sequence with a geometrically finite limit.

One can infer information about the volumes of convex cores of certain algebraically convergent sequences. Recall if $\Gamma$ is geometrically finite and $\Omega(\Gamma) \neq \emptyset$, then every finitely generated subgroup $\Gamma^{\prime} \subset \Gamma$ is itself geometrically finite [42]. Corollary 7.3 is an immediate consequence of both this fact and Theorem 7.2.

Corollary 7.3. Let $\left\{\varphi_{i}\right\}$ be a sequence of discrete faithful representations of a finitely generated torsion-free group $G$. Suppose the sequence is converging algebraically to a representation $\varphi_{\infty}$ so that $\Gamma=\varphi_{\infty}(G)$ is geometrically infinite. If $\left\{\Gamma_{i}=\varphi_{i}(G)\right\}$ has a geometrically convergent subsequence with a finitely generated limit $\Gamma_{G}$ so that $\Omega\left(\Gamma_{G}\right) \neq \emptyset$, then $\left\{\operatorname{vol} C\left(\Gamma_{i}\right)\right\}$ diverges.

\section{Remarks:}

1) It is possible to have $\lim \inf \operatorname{vol} C\left(\Gamma_{i}\right)>\operatorname{vol} C(\Gamma)$ under the lone assumption of geometric convergence of $\left\{\Gamma_{i}\right\}$ to $\Gamma$. Fix a basepoint in a hyperbolic manifold $M(\Gamma)$ not in the convex core and conjugate $\Gamma$ by a sequence Möbius transformations so the basepoint is traveling out an end of the manifold (relative to the convex core). One can verify using polyhedral convergence that the resulting sequence of Kleinian groups is converging geometrically to the identity, but the volumes of a $\epsilon$-neighborhood ( $\epsilon$ chosen arbitrarily) of the convex cores are a fixed value $C(\epsilon)>0$ in the sequence.

2) Theorem 7.2 has a spectral theory analogue.

Theorem. (Taylor [41] or [14]) Let $\left\{\Gamma_{i}\right\}$ be a sequence of discrete, torsionfree subgroups of Isom ${ }_{+}\left(\mathbf{H}^{n}\right)$, where $n \geq 3$. Suppose $\left\{\Gamma_{i}\right\}$ is converging geometrically to a discrete group $\Gamma_{\infty}$. Then

$$
\lim \sup \lambda_{0}\left(\Gamma_{i}\right) \leq \lambda_{0}\left(\Gamma_{\infty}\right)
$$


where $\lambda_{0}\left(\Gamma_{i}\right)$ (including $i=\infty$ ) is the infimum of the Rayleigh quotient over the space of smooth functions with compact support on $M\left(\Gamma_{i}\right)$ (see [40]).

We finish this section with the proof of continuity of volumes under strong convergence.

Theorem. Let $\left\{\varphi_{i}\right\}$ be a sequence of discrete faithful representations of $G$ into $P S L(2, \mathbf{C})$ converging strongly to a representation $\varphi_{\infty}$. Then

$$
\lim \operatorname{vol} C\left(\varphi_{i}(G)\right)=\operatorname{vol} C\left(\varphi_{\infty}(G)\right) .
$$

Proof. Recall that Theorem 7.2 allows us to restrict to the case that the limit group $\Gamma=\varphi_{\infty}(G)$ is geometrically finite.

Since $\Gamma$ is geometrically finite and $M(\Gamma)$ has infinite volume, then $\Omega(\Gamma) \neq$ $\emptyset$. Because the convergence of the representations is assumed to be strong, Theorem 4.8 in [24] implies that $\left\{L_{\Gamma_{i}}\right\}$ converges to $L_{\Gamma}$ in the Hausdorff set metric on $\widehat{\mathbf{C}}$. Fix a point $x_{\infty} \in M(\Gamma)$ and $0 \in \pi_{\infty}^{-1}\left(x_{\infty}\right)$.

We start with the case that the limit group is convex co-compact. In particular, we are in the situation described by Theorem 6.1: $\left\{\Gamma_{i}=\varphi_{i}(G)\right\}$ is a sequence of groups converging geometrically to a convex co-compact group $\Gamma$ and the sequence of limit sets $\left\{L_{\Gamma_{i}}\right\}$ is converging to $L_{\Gamma}$. Let the radial length $\tilde{r}$ be sufficiently large so that $\pi_{\infty}(B(0, \tilde{r})) \supset C(\Gamma)$. The proof of Theorem 6.1 demonstrates that for all index $i$ sufficiently large, the groups $\Gamma_{i}$ are convex co-compact, and in fact the lifts of the convex cores $\left\{\pi_{i}^{-1}\left(C\left(\Gamma_{i}\right)\right) \cap\right.$ $B(0, \tilde{r})\}$ are converging in the Hausdorff set metric to $\pi_{\infty}^{-1}(C(\Gamma)) \cap B(0, \tilde{r})$. The continuity of volume in this case follows immediately.

The first case we handle is that $s_{i}=s_{\infty}$. Choose $\epsilon>0$ so that $M^{(0, \epsilon]}\left(\Gamma_{i}\right)$ and $M^{(0, \epsilon]}(\Gamma)$ consist of $s_{\infty}$ cusps. Let $E_{\infty} \subset M^{(0, \epsilon]}(\Gamma)$ be a rank 1 cusp. Then by the assumptions of strong convergence and $s_{i}=s_{\infty}$, there exists a sequence of rank 1 cusps $E_{i} \subset M^{(0, \epsilon]}\left(\Gamma_{i}\right)$ so that $\left\{E_{i}\right\}$ is converging to $E_{\infty}$ quasi-isometrically on compact sets.

Let $K=\operatorname{vol} E_{\infty}$, and fix $\delta>0$. Then there exists a choice of injectivity radius $\epsilon^{\prime}<\epsilon$ so that vol $C^{\left[\epsilon^{\prime}, \infty\right)}(\Gamma) \cap E_{\infty}>K-\delta$. Because the convergence of $\left\{E_{i}\right\}$ to $E_{\infty}$ is $k_{i}$-quasi-isometric on compact sets with $k_{i} \rightarrow 1$, we observe that eventually

$$
\operatorname{vol}\left(C^{\left[\epsilon^{\prime}, \infty\right)}\left(\Gamma_{i}\right) \cap E_{i}\right)>K-\delta .
$$

Recall that any rank 1 cusp has a metric given by $d s^{2}=e^{-2 t}\left(d x^{2}+c l y^{2}\right)+d t^{2}$. Clearly Area $E_{i} \cap C^{\left(0, \epsilon^{\prime}\right]}\left(\Gamma_{i}\right)$ is converging to Area $E_{\infty} \cap C^{\left(0, \epsilon^{\prime}\right]}(\Gamma)$, so that 
eventually $\operatorname{vol}\left(E_{i} \cap C^{\left(0, \epsilon^{\prime}\right]}\left(\Gamma_{i}\right)\right)<K+\delta$ in the volume metric derived from $d s^{2}$. The rank 2 case is handled similar fashion. Showing the volumes of the cusps are converging handles the case when $s_{i}=s_{\infty}$, since vol $C^{\left[\epsilon^{\prime}, \infty\right)}\left(\Gamma_{i}\right)$ converges to $C^{\left[\epsilon^{\prime}, \infty\right)}(\Gamma) k_{i}$-quasi-isometrically. Each rank 1 and rank 2 cusp can be handled the same way (and there is a finite number of them), so the volume continuity follows in this case.

Recall Proposition 5.7; because of this fact we can suppose that $s_{i} \leq s<$ $s_{\infty}$, i.e. cusps are being formed in the limit (but none are "disappearing"). Because of the strong convergence, there exists a choice of injectivity radius $\epsilon$ so $C^{[\epsilon, \infty)}\left(\Gamma_{i}\right)$ is connected for all large index $i$. In effect, if $C^{[\epsilon, \infty)}\left(\Gamma_{i}\right)$ is not connected for all large index $i$ then some topology is being lost in the limit: we can prove this by using a geodesic argument similar to the one used in the Main Theorem.

As above, fix $\delta>0$. We consider a sequence of tubes $\left\{E_{i} \subset M^{(0, \epsilon)}\left(\Gamma_{i}\right)\right\}$ converging to a rank 1 cusp $E_{\infty} \subset M^{(0, \epsilon)}(\Gamma)$. Then there exists a choice of injectivity radius $\epsilon^{\prime}<\epsilon$ so that

$$
\operatorname{vol}\left(C^{\left[\epsilon^{\prime}, \infty\right)}\left(\Gamma_{i}\right) \cap E_{i}\right)>K-\delta
$$

where $K=\operatorname{vol} E_{\infty}$. To get the other inequality, first observe that the sequence $\left\{E_{i}\right\}$ is converging to a horoball fixed by the parabolic cyclic group $\left.<\varphi_{\infty}\right\rangle \subset \Gamma$ that uniformizes the cusp $E_{\infty}$ (see [10] section 3). Recall that each such rank 1 parabolic subgroup of $\Gamma$ is doubly cusped (see [32] Chapter VI), because $\Gamma$ is geometrically finite. Because the limit sets are converging, there exists constants $C_{i}$ so that

$$
\operatorname{vol}\left(E_{i} \cap C^{\left(0, \epsilon^{\prime}\right]}\left(\Gamma_{i}\right)\right) \leq C_{i} \operatorname{vol}\left(E_{\infty} \cap C^{\left(0, \epsilon^{\prime}\right]}(\Gamma)\right)
$$

and $C_{i} \rightarrow 1$. The result follows immediately.

Remark: The assumption of strong convergence is the lone assumption one can make on the convergence of the sequence of representations, and still get continuity of the volumes of the convex cores. For instance, a sequence of degenerate groups converging to a regular B-group on the boundary of a Bers' compactification provides an example of an algebraically convergent sequence where the volumes of the convex cores are not converging. Remark 1 following Corollary 7.3 gives a simple example of a geometrically convergent sequence so that the volumes of the convex cores are not converging. Example 6.2 demonstrates the assumption of geometric convergence and 
limit set convergence is not enough to show the volumes of the convex cores are continuous.

However, it might be true that if the sequence of representations is converging both algebraically and geometrically to different limits, then the volumes of the convex cores do exhibit some sort of convergence phenomena. Given the results of this section, we are motivated to make the following conjecture:

Conjecture 7.4. Let $G$ be an abstract, finitely generated torsion-free group. Let $\left\{\varphi_{i}\right\}$ be a sequence of discrete faithful representations of $G$ into $P S L(2, \mathbf{C})$, so that:

1. for each index $i$ the quotient manifold $\mathbf{H}^{3} / \Gamma_{i}$ has infinite volume,

2. $\left\{\varphi_{i}\right\}$ is converging algebraically to $\varphi_{\infty}$, and

3. $\left\{\Gamma_{i}\right\}$ is converging geometrically to a geometrically finite group $\Gamma$ and $\varphi_{\infty}(G)$ is properly contained in the geometric limit $\Gamma$.

Then

$$
\lim \operatorname{vol} C\left(\Gamma_{i}\right)=\operatorname{vol} C(\Gamma)
$$

and

$$
\lim \operatorname{vol} C\left(\Gamma_{i}\right)>\operatorname{vol} C\left(\varphi_{\infty}(G)\right) .
$$

We hope to return to this conjecture in a future note.

\section{References.}

[1] B. Abikoff, "On Boundaries of Teichmüller spaces and on Kleinian groups, III" Acta Math. 134(1975), pp. 211-237.

[2] A.F. Beardon, The Geometry of Discrete Groups, Springer-Verlag, 1983.

[3] R. Benedetti and C. Petronio, Lectures on Hyperbolic Geometry, SpringerVerlag, 1992.

[4] L. Bers, "On boundaries of Teichmüller spaces and on Kleinian groups," Ann. of Math. (2) 91(1970), pp. 570-600.

[5] L. Bers, "On moduli of Kleinian groups," Russian Math Surveys, 29(1974), pp. $88-102$.

[6] C. Bishop and P. Jones, "Hausdorff dimension and Kleinian groups", preprint. 
[7] F. Bonahon, "Bouts des variétés hyperboliques de dimension 3," Ann. of Math. 124(1986), pp. 71-158.

[8] B.H. Bowditch, "Geometrical finiteness for hyperbolic groups," J. Funct. Anal. 113(1993), pp. 245-317.

[9] B.H. Bowditch, "Some results on the geometry of convex hulls in manifolds of pinched negative curvature," Comment. Math. Helv. 69(1994), pp. 49-81.

[10] R. Brooks and J.P. Matelski, "Collars in Kleinian groups," Duke Math. J. 49(1982), pp. 163-182.

[11] R. Canary, D.B.A. Epstein and P. Green, "Notes on notes of Thurston," in Analytical and Geometrical Aspects of Hyperbolic Spaces, Cambridge University Press, 1987, pp. 3-92.

[12] R. Canary, "A covering theorem for hyperbolic 3-manifolds and its applications," Topology 35(1996), pp. 751-778.

[13] R. Canary and Y. Minsky, "On limits of tame hyperbolic 3-manifolds," J. Differential Geom. 43(1996), pp. 1-41.

[14] R. Canary, Y. Minsky and E. Taylor, "Spectral theory, Hausdorff dimension and the topology of hyperbolic 3-manifolds," J. Geom. Anal., to appear.

[15] A. Douady and C. Earle, "Conformally natural extensions of homeomorphisms of the circle, "Acta Math. 157(1986), pp. 23-48.

[16] C. Earle and A. Marden, "Quasifuchsian space and geometric complex cöordinates for Teichmüller and moduli space,"preprint.

[17] D.B.A. Epstein et al., Word Processing in Groups, Jones and Bartlett, 1992.

[18] D.B.A. Epstein and A. Marden, "Convex hulls in hyperbolic space, a theorem of Sullivan, and measured pleated surfaces," in Analytical and Geometrical Aspects of Hyperbolic Spaces, Cambridge University Press, 1987, pp. 114-253.

[19] K.J. Falconer, The Geometry of Fractal Sets, Cambridge University Press, 1985.

[20] F. Gehring and J. Väisälä, "Hausdorff dimension and quasi-conformal mappings," J. London Math. Soc. (2) 6(1973) pp. 504-512.

[21] L. Greenberg, "Fundamental polyhedron for Kleinian groups, "Ann. of Math. 84(1966), pp. 433-441.

[22] T. Jørgensen, "On cyclic groups of Möbius transformations, "Math. Scand. 33(1973), pp. 250-260. 
[23] T. Jørgensen, "On discrete groups of Möbius transformations, "Amer. J. Math. 98(1976), pp. 739-749.

[24] T. Jørgensen and A. Marden, "Algebraic and geometric convergence of Kleinian groups," Math. Scand. 66(1990), pp. 47-72.

[25] L. Keen, B. Maskit and C. Series, "Geometric finiteness and uniqueness for Kleinian groups with circle packing limit sets," J. reine angew. Math. 436(1993), pp. 209-219.

[26] S. Kerckhoff and W. Thurston, "Non-continuity of the modular group at Bers' boundary of Teichüller space," Invent. Math. 100 (1990), pp. 25-47.

[27] A. Marden, "The geometry of finitely generated Kleinian groups," Ann. of Math. 99(1974), pp. 383-462.

[28] A. Marden, "Geometric relations between homeomorphic Riemann surfaces," Bull. A.M.S. 3(1980), pp. 1001-1017.

[29] A. Marden, personal communication at Gehring Conference, 1995.

[30] G.A. Margulis, "Arithmeticity of nonuniform lattices," Funkcional Anal. Prilžen. 7(1973), pp. 88-89.

[31] B. Maskit, "On boundaries of Teichmüller spaces and on Kleinian groups," Ann. of Math. 91(1970), pp. 607-639.

[32] B. Maskit, Kleinian Groups, Springer-Verlag, 1988.

[33] C. McMullen, "Cusps are dense," Ann. of Math. 133(1991), pp. 217-247.

[34] J. Morgan, "Uniformization theorem for three-dimensional manifolds," in The Smith Conjecture, Academic Press, 1984, pp. 37-125.

[35] G.D. Mostow, Quasi-conformal mappings in $n$-space and the rigidity of hyperbolic space forms, Inst. Hautes. Études Sci. Publ. Math. 34(1968), pp. 53-104.

[36] H.M. Reimann, "Invariant extension of quasi-conformal deformations," $A n n$. Acad. Sci. Fenn. Ser. A I Math., 20 (1985), pp. 477-492.

[37] P. Scott, "Compact submanifolds of 3-manifolds," J. London Math. Soc. 7(1973), pp. 246-250.

[38] D. Sullivan," A finiteness theorem for cusps," Acta Math. 147(1981), pp. 289299.

[39] D. Sullivan, "Entropy, Hausdorff measures old and new, and limit sets of geometrically finite Kleinian groups," Acta Math. 153 (1984), pp. 259-277. 
[40] D. Sullivan, "Related aspects of positivity in Riemannian geometry," J. Differential Geom. 25(1987), pp. 327-351.

[41] E.C. Taylor, "Spectral theory and the convergence of Kleinian groups,"preprint.

[42] W. Thurston, The Geometry and Topology of 3-manifolds, Lecture Notes, Department of Mathematics, Princeton University, Princeton 1979.

[43] P. Tukia, "The Hausdorff dimension of the limit set of a geometrically finite Kleinian group," Acta Math. 152(1984), pp. 127-180.

RECEIVED MAY 16, 1996.

DEPARTMENT OF MATHEMATICS

UNIVERSITY OF MICHIGAN

ANN ARBOR, MI 48109 